\title{
Pulsed squeezed-light generation in a waveguide with second-subharmonic generation and periodic corrugation
}

\author{
Jan Peřina Jr. \\ RCPTM, Joint Laboratory of Optics of Palacky University and \\ Institute of Physics of Academy of Sciences of the Czech Republic, \\ 17. listopadu 12, 77146 Olomouc, Czech Republif
}

\begin{abstract}
Quantum pulsed second-subharmonic generation in a planar waveguide with a small periodic corrugation at the surface is studied. Back-scattering of the interacting fields on the corrugation enhances the nonlinear interaction giving larger values of squeezing. The problem of back-scattering is treated by perturbation theory, using the Fourier transform for non-dispersion propagation, and by numerical approach in the general case. Optimum spectral modes for squeezed-light generation are found using the Bloch-Messiah reduction. Improvement in squeezing and increase of numbers of generated photons are quantified for the corrugation resonating with the fundamental and secondsubharmonic field. Splitting of the generated pulse by the corrugation is predicted.
\end{abstract}

PACS numbers: 42.50.Dv,42.50.Lc,42.65.Yj,42.82.Et

\section{INTRODUCTION}

The process of second-subharmonic generation which is just the inverse process to that of second-harmonic generation [1] is interesting not only as a means of frequency conversion. It can also serve as an efficient source of squeezed light 2] which amplitude fluctuations are suppressed below the limit given by quantum uncertainty relations (for a review, see, e.g., [3 [- 6 ]). Such nonclassical light can be emitted both in the fundamental as well as second-subharmonic frequency (SSF) fields 7]. Also light with nonclassical photon-number statistics can be obtained in this process under suitable conditions [8, 9]. The process of second-subharmonic generation belongs to the whole family of optical parametric processes which share many common features [10-12]. Among them, spontaneous parametric down-conversion with its ability to generate entangled photon pairs plays an important role [4].

In homogeneous nonlinear media, the perfectly phase matched nonlinear interaction gives the largest possible squeezing of amplitude fluctuations. The principal squeeze variance of the SSF field asymptotically reaches zero for large values of the gain of nonlinear interaction [13]. That is why enhancement of the effective nonlinearity is important as it immediately results in larger squeezing and lower pumping intensities needed for reaching a given value of squeezing. For this purpose, configurations with cavities filled by a nonlinear medium have been usually used to generate squeezed light (e.g., 7, 14]). Also nonlinear waveguides, for which strong spatial localization of optical fields in the transverse plane is characteristic, profit from enhancement of the effective nonlinearity. This enhancement has been widely exploited when generating photon pairs in classical [15 17], multi-

*Electronic address: perinaj@prfnw.upol.cz layer [18], Bragg-reflection [19, 20] and photonic-wire 21] waveguides. Effective nonlinearity in a waveguide can also be increased by the use of mode coupling through evanescent waves with fields in a neighboring waveguide. This occurs due to an additional spatial modulation induced by energy exchange [22, 23]. Additional spatial modulation can also be introduced in a simpler geometry using a linear periodic corrugation at or below the waveguide surface [24, 25]. Back-scattering occurring at the corrugation modifies electric-field amplitudes of the nonlinearly interacting fields which results in the enhancement of effective nonlinearity under suitable conditions $26-28]$. Back-scattering on a periodic corrugation has already been exploited for $\mathrm{cw}$ squeezed-light generation both in the process of second-harmonic [29] and second-subharmonic generation 28]. Scattering on a periodic corrugation in a waveguide can also be used to enhance second-harmonic generation in Čerenkov configuration 30 32]. Squeezed-light generation in nonlinear photonic structures has been discussed in general in [33].

Photonic structures modify in general phase matching conditions of the nonlinear interaction. Propagation constants in waveguiding structures represent a typical example. Especially Bragg-reflection waveguides offer wide possibilities in this direction [34, 35]. Also scattering on a periodic corrugation gives an additional term to nonlinear phase-matching conditions [36 38]. For this reason we need a tool that allows us to reach nonlinear phase matching conditions for an arbitrary photonic structure. Periodic poling 39 42] of $\chi^{(2)}$ susceptibility has occurred to be extraordinarily useful here and has resulted in the so-called quasi-phase-matched nonlinear interactions. Using this method even spectrally broadband two-mode nonlinear interaction with femtosecond pulses has become possible [43].

Due to temporal energy concentration, the pulsed regime of the nonlinear process allows to use lower pumping powers to observe the needed level of squeezing. It also brings into attention new features of the generated light, namely its spectral modal structure. 
In the pulsed regime and travelling-wave configuration, squeezed-light generation in the considered nonlinear interaction has been studied with the help of phasespace quasi-distributions or the corresponding Langevin stochastic equations [44]. A local oscillator in the form of an ultrashort optical pulse is needed to observe pulsed squeezing experimentally using homodyne detection. The effort to observe the largest possible values of squeezing has raised the question about an optimum shape of the local-oscillator field [45 47]. The BlochMessiah reduction of the evolution matrices (operators) has been proposed for the solution of the modal structure [48]. This method has been elaborated in detail in [49, 50] for degenerate parametric down-conversion in a $\mathrm{BBO}$ crystal. Also the relation between the Bloch-Messiah reduction and Schmidt decomposition of a two-photon spectral amplitude characterizing spontaneous parametric down-conversion has been found. The results obtained in single-pass geometry have been generalized to the nonlinear interaction in a cavity [51]. Mode structure of nonclassical states arising from squeezed states after post-selection done with on/off detectors has been analyzed in 52]. The discussed effects occurring in temporal (or spectral) domains have their counterparts in spatial domain, in the transverse plane of the beams. Also here eigenmodes typical for the nonlinear interaction can be revealed [53]. Circular symmetry of the usual optical beams, however, results in different types of eigenmodes defined in the transverse plane. Despite this, a close similarity in the behavior of fields in the spectral and spatial domains can be found. As an important example, correlations between intensities of the interacting fields in their transverse planes can be mentioned [54, 55].

Here, continuing the investigation in [28] we pay attention to the pulsed SSF generation in a waveguide with linear periodic corrugation on its surface that causes back-scattering of the interacting fields. Assuming strong pulsed fundamental field, we study squeezed-light generation in the SSF field. We pay our attention to the enhancement of nonlinearity and the related increase of squeezing applying three different approaches. We utilize a sophisticated perturbation approach, the method of Fourier transformation in non-dispersion field propagation, and numerical solution for the general case. Squeezing is characterized by a principal squeeze variance introduced in [2, 3, 5] that is determined for suitable spectral modes.

The paper is organized as follows. In Sec. II, a multimode quantum model of the nonlinear interaction using the appropriate momentum operator and the related Heisenberg equations is presented. Sec. III is devoted to the perturbation solution of the model that is divided into three parts. A general perturbation solution is found in Subsec. IIIA, Gaussian spectral approximation to the solution is applied in Subsec. IIIB, and squeezing is analyzed in Subsec. IIIC. If inter-mode dispersion is omitted, the model can be solved by the Fourier transform, as shown in Sec. IV. Discrete formulation of the model is elaborated in Sec. V devoted to the numerical solution. Also quantities useful in the characterization of the generated field are introduced in this section. Discussion of squeezing and appropriate spectral eigenmodes is contained in Sec. VI. Whereas the model with non-dispersion field propagation is analyzed in Subsec. VIA, the results of the general approach are studied in Subsec. VIB. Conclusions are drawn in Sec. VII. An optimum mode profile giving the maximum pulsed squeezing is found in Appendix A.

\section{QUANTUM MULTI-MODE MODEL OF SECOND-SUBHARMONIC GENERATION}

We consider a nonlinear waveguide made of $\mathrm{LiNbO}_{3}$ (see Fig. 11) in the configuration that allows to generate a second-subharmonic (SSF) field using $\chi^{(2)}$ nonlinearity and pumping, e.g., by the second-harmonic of a Nd:YAG laser at the wavelength of $\lambda_{s}=1.064 \times 10^{-6} \mathrm{~m}$. Under a suitable choice of waveguide parameters, the waveguide is single-mode for both the fundamental and SSF field and allows for efficient nonlinear interaction. A linear corrugation fabricated at the top of the waveguide leads to back-scattering of the interacting fields which results in the enhancement of electric-field amplitudes inside the waveguide under suitable conditions. This leads to effective increase of the nonlinear interaction and gives larger amount of squeezing of the SSF light. Quasi-phase matching of the nonlinear interaction is guaranteed by periodic poling with an appropriate poling period. A detailed description of the waveguide was given in [28]. It has been shown that the investigated waveguide can be described by the following parameters: propagation and coupling constants of the fundamental and SSF fields and constants characterizing the nonlinear interaction occurring among both the forward- and backward-propagating fields. When losses inside the waveguide caused both by absorption and scattering of the light outside the guided modes are neglected we can describe the nonlinear interaction in the waveguide as follows.

According to quantum theory, the electric-field vector operator amplitudes $\hat{\mathbf{E}}_{a}(x, y, z, t)(a=p, s)$ at time $t$ and spatial point $(x, y, z)$ inside the waveguide can be decomposed into harmonic plane waves with mode operator amplitudes $\hat{a}_{a}$ in the Heisenberg picture [6, 9] :

$$
\begin{aligned}
& \hat{\mathbf{E}}_{a}(x, y, z, t)=\int_{0}^{\infty} d \omega_{a} \hat{\mathbf{E}}_{a}\left(x, y, z, \omega_{a}\right) \exp \left(-i \omega_{a} t\right), \\
& \hat{\mathbf{E}}_{a}\left(x, y, z, \omega_{a}\right)=i\left[\hat{a}_{a_{F}}\left(z, \omega_{a}\right) \mathbf{e}_{a}\left(x, y, \omega_{a}\right)\right. \\
& \left.\quad+\hat{a}_{a_{B}}\left(z, \omega_{a}\right) \mathbf{e}_{a}\left(x, y, \omega_{a}\right)-\text { H.c. }\right], \quad a=p, s
\end{aligned}
$$

In Eq. (2), $\hat{a}_{a_{F}}\left(z, \omega_{a}\right)\left[\hat{a}_{a_{B}}\left(z, \omega_{a}\right)\right]$ denotes an annihilation operator of the mode with frequency $\omega_{a}$ in field $a$ propagating forward [backward]. We note that the mode vector functions $\mathbf{e}_{a}$ as well as the corresponding propagation constants $\beta_{a}$ depend on the frequency $\omega_{a}$ and their 


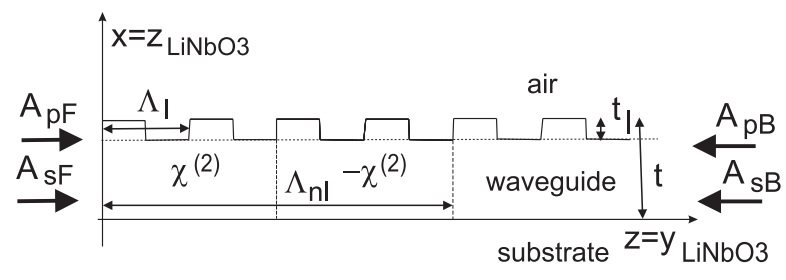

FIG. 1: Scheme of a periodically-poled nonlinear waveguide with a rectangular transverse profile (thickness $t$ and width $\Delta y$ ) and length $L$ having $\chi^{(2)}$ susceptibility. A linear corrugation with period $\Lambda_{l}$ and depth $t_{l}$ occurs on the waveguide upper surface; $\Lambda_{n l}$ denotes period of nonlinear poling. The waveguide is made of $\mathrm{LiNbO}_{3}$ which optical axis coincides with the $x$ axis. Four fields interact inside the waveguide: forward-propagating fundamental (electricfield amplitude $A_{p_{F}}$ ), backward-propagating fundamental $\left(A_{p_{B}}\right)$, forward-propagating second-subharmonic $\left(A_{s_{F}}\right)$, and backward-propagating second-subharmonic $\left(A_{s_{B}}\right)$ fields.

form can be found in 28].

Evolution of the nonlinearly-interacting quantum optical fields inside the waveguide is described by the following momentum operator $\hat{G}[9,23]$ :

$$
\begin{aligned}
& \hat{G}(z)= \\
& \sum_{a=p, s} \sum_{b=F, B} \int_{0}^{\infty} d \omega_{a}( \pm 1)_{b} \hbar \beta_{a_{b}}\left(\omega_{a}\right) \hat{a}_{a_{b}}^{\dagger}\left(z, \omega_{a}\right) \hat{a}_{a_{b}}\left(z, \omega_{a}\right) \\
& +\left[\sum_{a=p, s} \int_{0}^{\infty} d \omega_{a} \hbar K_{a}\left(\omega_{a}\right) \exp \left(i \frac{2 \pi}{\Lambda_{a}} z\right)\right. \\
& \left.\quad \times \hat{a}_{a_{F}}^{\dagger}\left(z, \omega_{a}\right) \hat{a}_{a_{B}}\left(z, \omega_{a}\right)+\text { H.c. }\right] \\
& -\left[\sum_{b=F, B} 2 i \int_{0}^{\infty} d \omega_{s} \int_{0}^{\infty} d \omega_{s}^{\prime}( \pm 1)_{b} K_{n l, q}\left(\omega_{s}, \omega_{s}^{\prime}\right)\right. \\
& \quad \times \exp \left(i \frac{2 \pi q}{\Lambda_{n l}} z\right) \hat{a}_{s_{b}}^{\dagger}\left(z, \omega_{s}\right) \hat{a}_{s_{b}}^{\dagger}\left(z, \omega_{s}^{\prime}\right) \hat{a}_{p_{b}}\left(z, \omega_{s}+\omega_{s}^{\prime}\right) \\
& \quad+\text { H.c. }] ;
\end{aligned}
$$

$( \pm 1)_{F} \equiv 1$ and $( \pm 1)_{B} \equiv-1$. The linear coupling constants $K_{p}\left(\omega_{p}\right)\left[K_{s}\left(\omega_{s}\right)\right]$ describe scattering on the corrugation with period $\Lambda_{p}\left[\Lambda_{s}\right]$ that leads to the coupling between the fundamental [SSF] fields propagating forward and backward. Under suitable conditions, this scattering leads to increased values of electric-field amplitudes inside the waveguide. However, this increase is much lower than that found in layered photonic band-gap structures [56 59]. On the other hand, the nonlinear coupling constants $K_{n l, q}\left(\omega_{s}, \omega_{s}^{\prime}\right)$ characterize the nonlinear interaction among co-propagating fields and obey the symmetry relation $K_{n l, q}\left(\omega_{s}, \omega_{s}^{\prime}\right)=K_{n l, q}\left(\omega_{s}^{\prime}, \omega_{s}\right)$. Constant $\Lambda_{n l}$ gives the poling period whereas the integer number $q$ determines a harmonic frequency employed in the quasiphase-matching. Planck constant is denoted as $\hbar$ and symbol H.c. substitutes the Hermitian conjugated term.

The Heisenberg equations, $d \hat{X} / d z=-i / \hbar[\hat{G}, \hat{X}]$ for an arbitrary operator $\hat{X}$, written for the momentum operator $\hat{G}$ in Eq. (3) attain the form:

$$
\begin{aligned}
& \frac{d \hat{a}_{s_{F}}\left(z, \omega_{s}\right)}{d z}=i \beta_{s}\left(\omega_{s}\right) \hat{a}_{s_{F}}\left(z, \omega_{s}\right) \\
& +i K_{s}\left(\omega_{s}\right) \exp \left(i \frac{2 \pi}{\Lambda_{s}} z\right) \hat{a}_{s_{B}}\left(z, \omega_{s}\right) \\
& +4 \int_{0}^{\infty} d \omega_{s}^{\prime} K_{n l, q}\left(\omega_{s}, \omega_{s}^{\prime}\right) \exp \left(i \frac{2 \pi q}{\Lambda_{n l}} z\right) \\
& \times \hat{a}_{p_{F}}\left(z, \omega_{s}+\omega_{s}^{\prime}\right) \hat{a}_{s_{F}}^{\dagger}\left(z, \omega_{s}^{\prime}\right), \\
& \frac{d \hat{a}_{s_{B}}\left(z, \omega_{s}\right)}{d z}=-i \beta_{s}\left(\omega_{s}\right) \hat{a}_{s_{B}}\left(z, \omega_{s}\right) \\
& -i K_{s}^{*}\left(\omega_{s}\right) \exp \left(-i \frac{2 \pi}{\Lambda_{s}} z\right) \hat{a}_{s_{F}}\left(z, \omega_{s}\right) \\
& -4 \int_{0}^{\infty} d \omega_{s}^{\prime} K_{n l, q}\left(\omega_{s}, \omega_{s}^{\prime}\right) \exp \left(i \frac{2 \pi q}{\Lambda_{n l}} z\right) \\
& \times \hat{a}_{p_{B}}\left(z, \omega_{s}+\omega_{s}^{\prime}\right) \hat{a}_{s_{B}}^{\dagger}\left(z, \omega_{s}^{\prime}\right), \\
& \frac{d \hat{a}_{p_{F}}\left(z, \omega_{p}\right)}{d z}=i \beta_{p}\left(\omega_{p}\right) \hat{a}_{p_{F}}\left(z, \omega_{p}\right) \\
& +i K_{p}\left(\omega_{p}\right) \exp \left(i \frac{2 \pi}{\Lambda_{p}} z\right) \hat{a}_{p_{B}}\left(z, \omega_{p}\right) \\
& -2 \int_{0}^{\infty} d \omega_{s} K_{n l, q}^{*}\left(\omega_{s}, \omega_{p}-\omega_{s}\right) \exp \left(-i \frac{2 \pi q}{\Lambda_{n l}} z\right) \\
& \times \hat{a}_{s_{F}}\left(z, \omega_{s}\right) \hat{a}_{s_{F}}\left(z, \omega_{p}-\omega_{s}\right), \\
& \frac{d \hat{a}_{p_{B}}\left(z, \omega_{p}\right)}{d z}=-i \beta_{p}\left(\omega_{p}\right) \hat{a}_{p_{B}}\left(z, \omega_{p}\right) \\
& -i K_{p}^{*}\left(\omega_{p}\right) \exp \left(-i \frac{2 \pi}{\Lambda_{p}} z\right) \hat{a}_{p_{F}}\left(z, \omega_{p}\right) \\
& +2 \int_{0}^{\infty} d \omega_{s} K_{n l, q}^{*}\left(\omega_{s}, \omega_{p}-\omega_{s}\right) \exp \left(-i \frac{2 \pi q}{\Lambda_{n l}} z\right) \\
& \times \hat{a}_{s_{B}}\left(z, \omega_{s}\right) \hat{a}_{s_{B}}\left(z, \omega_{p}-\omega_{s}\right) .
\end{aligned}
$$

Creation and annihilation operators of the incident fields are assumed to fulfil the boson commutation relations, i.e.

$$
\begin{gathered}
{\left[\hat{a}_{a_{F}}\left(0, \omega_{a}\right), \hat{a}_{a_{F}^{\prime}}^{\dagger}\left(0, \omega_{a^{\prime}}\right)\right]=\delta_{a, a^{\prime}} \delta\left(\omega_{a}-\omega_{a^{\prime}}\right),} \\
{\left[\hat{a}_{a_{B}}\left(L, \omega_{a}\right), \hat{a}_{a_{B}^{\prime}}^{\dagger}\left(L, \omega_{a^{\prime}}\right)\right]=\delta_{a, a^{\prime}} \delta\left(\omega_{a}-\omega_{a^{\prime}}\right),} \\
a=p, s .
\end{gathered}
$$

The remaining commutators are zero. It has been shown in [60] for quadratic momentum operators $\hat{G}$ that also the output operators obey the boson commutation relations:

$$
\begin{gathered}
{\left[\hat{a}_{a_{F}}\left(L, \omega_{a}\right), \hat{a}_{a_{F}^{\prime}}^{\dagger}\left(L, \omega_{a^{\prime}}\right)\right]=\delta_{a, a^{\prime}} \delta\left(\omega_{a}-\omega_{a^{\prime}}\right),} \\
{\left[\hat{a}_{a_{B}}\left(0, \omega_{a}\right), \hat{a}_{a_{B}^{\prime}}^{\dagger}\left(0, \omega_{a^{\prime}}\right)\right]=\delta_{a, a^{\prime}} \delta\left(\omega_{a}-\omega_{a^{\prime}}\right),} \\
a=p, s
\end{gathered}
$$

and commutators not mentioned in Eq. (6) are zero.

We note that the nonlinear operator equations written in Eq. (4) have one integral of motion arising from the 
conservation of energy flux:

$$
\begin{aligned}
\frac{d}{d z} & {\left[\int_{0}^{\infty} d \omega_{s} \hat{a}_{s_{F}}^{\dagger}\left(z, \omega_{s}\right) \hat{a}_{s_{F}}\left(z, \omega_{s}\right)\right.} \\
& -\int_{0}^{\infty} d \omega_{s} \hat{a}_{s_{B}}^{\dagger}\left(z, \omega_{s}\right) \hat{a}_{s_{B}}\left(z, \omega_{s}\right) \\
& +2 \int_{0}^{\infty} d \omega_{p} \hat{a}_{p_{F}}^{\dagger}\left(z, \omega_{p}\right) \hat{a}_{p_{F}}\left(z, \omega_{p}\right) \\
& \left.-2 \int_{0}^{\infty} d \omega_{p} \hat{a}_{p_{B}}^{\dagger}\left(z, \omega_{p}\right) \hat{a}_{p_{B}}\left(z, \omega_{p}\right)\right]=0 .
\end{aligned}
$$

It is convenient to introduce new operators $\hat{A}$ that take into account the harmonic spatial evolution induced by the corrugation present in general in both the fundamental and SSF fields, $\hat{a}_{a_{F}}\left(z, \omega_{a}\right)=\hat{A}_{a_{F}}\left(z, \omega_{a}\right) \exp \left(i \pi z / \Lambda_{a}\right)$ and $\hat{a}_{a_{B}}\left(z, \omega_{a}\right)=\hat{A}_{a_{B}}\left(z, \omega_{a}\right) \exp \left(-i \pi z / \Lambda_{a}\right)$ for $a=p, s$. Equations (4) written for the operators $\hat{A}$ take the form:

$$
\begin{aligned}
\frac{d \hat{A}_{s_{F}}\left(z, \omega_{s}\right)}{d z}= & i \frac{\delta_{s}\left(\omega_{s}\right)}{2} \hat{A}_{s_{F}}\left(z, \omega_{s}\right)+i K_{s}\left(\omega_{s}\right) \hat{A}_{s_{B}}\left(z, \omega_{s}\right) \\
+4 \int_{0}^{\infty} & d \omega_{s}^{\prime} K_{n l, q}\left(\omega_{s}, \omega_{s}^{\prime}\right) \exp \left[i \delta_{n l, q} z\right] \\
& \times \hat{A}_{p_{F}}\left(z, \omega_{s}+\omega_{s}^{\prime}\right) \hat{A}_{s_{F}}^{\dagger}\left(z, \omega_{s}^{\prime}\right), \\
\frac{d \hat{A}_{s_{B}}\left(z, \omega_{s}\right)}{d z}= & -i \frac{\delta_{s}\left(\omega_{s}\right)}{2} \hat{A}_{s_{B}}\left(z, \omega_{s}\right)-i K_{s}^{*}\left(\omega_{s}\right) \hat{A}_{s_{F}}\left(z, \omega_{s}\right) \\
-4 \int_{0}^{\infty} & d \omega_{s}^{\prime} K_{n l, q}\left(\omega_{s}, \omega_{s}^{\prime}\right) \exp \left[-i \delta_{n l, q} z\right] \\
\times & \hat{A}_{p_{B}}\left(z, \omega_{s}+\omega_{s}^{\prime}\right) \hat{A}_{s_{B}}^{\dagger}\left(z, \omega_{s}^{\prime}\right), \\
\frac{d \hat{A}_{p_{F}}\left(z, \omega_{p}\right)}{d z} & i \frac{\delta_{p}\left(\omega_{p}\right)}{2} \hat{A}_{p_{F}}\left(z, \omega_{p}\right)+i K_{p}\left(\omega_{p}\right) \hat{A}_{p_{B}}\left(z, \omega_{p}\right) \\
-2 \int_{0}^{\infty} & d \omega_{s} K_{n l, q}^{*}\left(\omega_{s}, \omega_{p}-\omega_{s}\right) \exp \left[-i \delta_{n l, q} z\right] \\
\times & \hat{A}_{s_{F}}\left(z, \omega_{s}\right) \hat{A}_{s_{F}}\left(z, \omega_{p}-\omega_{s}\right), \\
= & -i \frac{\delta_{p}\left(\omega_{p}\right)}{2} \hat{A}_{p_{B}}\left(z, \omega_{p}\right)-i K_{p}^{*}\left(\omega_{p}\right) \hat{A}_{p_{F}}\left(z, \omega_{p}\right) \\
\frac{d \hat{A}_{p_{B}}\left(z, \omega_{p}\right)}{d z} & \times \hat{A}_{s_{B}}\left(z, \omega_{s}\right) \hat{A}_{s_{B}}\left(z, \omega_{p}-\omega_{s}\right) . \\
+2 \int_{0}^{\infty} & d \omega_{s} K_{n l, q}^{*}\left(\omega_{s}, \omega_{p}-\omega_{s}\right) \exp \left[i \delta_{n l, q} z\right]
\end{aligned}
$$

Linear phase mismatches $\delta_{p}, \delta_{s}$ and nonlinear phase mismatch $\delta_{n l, q}(q=0, \pm 1)$ are defined as:

$$
\begin{aligned}
\delta_{a}\left(\omega_{a}\right) & =2 \beta_{a}\left(\omega_{a}\right)-\frac{2 \pi}{\Lambda_{a}}, \quad a=p, s \\
\delta_{n l, q} & =\frac{\pi}{\Lambda_{p}}-2 \frac{\pi}{\Lambda_{s}}+\frac{2 \pi q}{\Lambda_{n l}} .
\end{aligned}
$$

We note that whereas $q$ was used for the nonlinear interaction among the forward-propagating fields, $-q$ was chosen for the interaction among the backward-propagating fields in Eqs. (8) and (9). We also note that equations similar to (8) and (9) can be derived from the wave equation considering classical fields and invoking paraxial approximation [12].
We assume that the fundamental field is strong and its depletion due to the interaction with the SSF field can be neglected. In this case, the equations in (9) become linear and break into groups with two linear equations for a given frequency $\omega_{p}$ :

$$
\begin{aligned}
& \frac{d \hat{A}_{p_{F}}\left(z, \omega_{p}\right)}{d z}=i \frac{\delta_{p}\left(\omega_{p}\right)}{2} \hat{A}_{p_{F}}\left(z, \omega_{p}\right)+i K_{p}\left(\omega_{p}\right) \hat{A}_{p_{B}}\left(z, \omega_{p}\right), \\
& \frac{d \hat{A}_{p_{B}}\left(z, \omega_{p}\right)}{d z}=-i \frac{\delta_{p}\left(\omega_{p}\right)}{2} \hat{A}_{p_{B}}\left(z, \omega_{p}\right)-i K_{p}^{*}\left(\omega_{p}\right) \hat{A}_{p_{F}}\left(z, \omega_{p}\right) .
\end{aligned}
$$

The solution of Eqs. (11) can be easily obtained in the matrix form

$$
\begin{aligned}
{\left[\begin{array}{c}
\hat{A}_{p_{F}}\left(z, \omega_{p}\right) \\
\hat{A}_{p_{B}}\left(z, \omega_{p}\right)
\end{array}\right]=} & \sum_{ \pm} B_{p}^{ \pm}\left(\omega_{p}\right) \exp \left[ \pm i \Delta_{p}\left(\omega_{p}\right) z\right] \\
& \times\left[\begin{array}{c}
\hat{A}_{p_{F}}\left(0, \omega_{p}\right) \\
\hat{A}_{p_{B}}\left(L, \omega_{p}\right)
\end{array}\right]
\end{aligned}
$$

using the eigenfrequencies $\pm \Delta_{p}, \quad \Delta_{p}\left(\omega_{p}\right)=$ $\sqrt{\delta_{p}^{2}\left(\omega_{p}\right) / 4-\left|K_{p}\left(\omega_{p}\right)\right|^{2}}$. Symbol $\sum_{ \pm}$occurring in Eq. (12) means the summation over the terms differing in their signs. We note that the boundary condition for the backward-propagating field is chosen at $z=L$. The matrices $B_{p}^{ \pm}\left(\omega_{p}\right)$ are defined as

$$
B_{p}^{ \pm}\left(\omega_{p}\right)=D_{p}\left[\begin{array}{cc}
\left( \pm \frac{\delta_{p}}{2}+\Delta_{p}\right) \exp \left(\mp i \Delta_{p} L\right) & \pm K_{p} \\
\mp K_{p}^{*} \exp \left(\mp i \Delta_{p} L\right) & \mp \frac{\delta_{p}}{2}+\Delta_{p}
\end{array}\right]
$$

and $D_{p}\left(\omega_{p}\right)=\left[2 \Delta_{p} \cos \left(\Delta_{p} L\right)-i \delta_{p} \sin \left(\Delta_{p} L\right)\right]^{-1}$.

If the corrugation is missing, the matrices $B_{p}^{ \pm}$in Eq. (13) take a simple form obtainable in the limit $K_{p} \longrightarrow 0$ together with $\Lambda_{p} \longrightarrow \infty$ :

$$
B_{p}^{+}=\left[\begin{array}{ll}
1 & 0 \\
0 & 0
\end{array}\right], \quad B_{p}^{-}=\left[\begin{array}{cc}
0 & 0 \\
0 & \exp \left(i \beta_{p} L\right)
\end{array}\right]
$$

It also holds that $\delta_{p}=2 \beta_{p}, \Delta_{p}=\beta_{p}$ and $D_{p}=$ $\exp \left(i \beta_{p} L\right) /\left(2 \beta_{p}\right)$ in this limit.

\section{PERTURBATION SOLUTION}

Perturbation solution of two equations (8) can be found after substituting the solution for the fundamental field contained in Eq. (12). In the perturbation approach, the inquired solution $\hat{A}_{s_{b}}\left(z, \omega_{s}\right)$ for $b=F, B$ is expressed as $\sum_{n=0}^{\infty} \hat{A}_{s_{b}}^{(n)}\left(z, \omega_{s}\right)$ where an $n$-th term $\hat{A}_{s_{b}}^{(n)}\left(z, \omega_{s}\right)$ is proportional to $K_{n l, q}^{n}$. 


\section{A. General solution up to the first order in nonlinearity}

The zeroth-order terms $\hat{A}_{s_{F}}^{(0)}\left(z, \omega_{s}\right)$ and $\hat{A}_{s_{B}}^{(0)}\left(z, \omega_{s}\right)$ are given as a solution to the equations

$\frac{d \hat{A}_{s_{F}}^{(0)}\left(z, \omega_{s}\right)}{d z}=i \frac{\delta_{s}\left(\omega_{s}\right)}{2} \hat{A}_{s_{F}}^{(0)}\left(z, \omega_{s}\right)+i K_{s}\left(\omega_{s}\right) \hat{A}_{s_{B}}^{(0)}\left(z, \omega_{s}\right)$, $\frac{d \hat{A}_{s_{B}}^{(0)}\left(z, \omega_{s}\right)}{d z}=-i \frac{\delta_{s}\left(\omega_{s}\right)}{2} \hat{A}_{s_{B}}^{(0)}\left(z, \omega_{s}\right)-i K_{s}^{*}\left(\omega_{s}\right) \hat{A}_{s_{F}}^{(0)}\left(z, \omega_{s}\right)$.

This solution can be written in the compact matrix form:

$$
\begin{aligned}
{\left[\begin{array}{c}
\hat{A}_{s_{F}}\left(z, \omega_{s}\right) \\
\hat{A}_{s_{B}}\left(z, \omega_{s}\right)
\end{array}\right]=} & \sum_{ \pm} \tilde{B}_{s}^{ \pm}\left(\omega_{s}\right) \exp \left[ \pm i \Delta_{s}\left(\omega_{s}\right) z\right] \\
& \times\left[\begin{array}{c}
\hat{A}_{s_{F}}\left(0, \omega_{s}\right) \\
\hat{A}_{s_{B}}\left(0, \omega_{s}\right)
\end{array}\right]
\end{aligned}
$$

$\Delta_{s}\left(\omega_{s}\right)=\sqrt{\delta_{s}^{2}\left(\omega_{s}\right) / 4-\left|K_{s}\left(\omega_{s}\right)\right|^{2}}$. The matrices $\tilde{B}_{s}^{ \pm}\left(\omega_{s}\right)$ are expressed as

$$
\tilde{B}_{s}^{ \pm}\left(\omega_{s}\right)=\frac{1}{2 \Delta_{s}}\left[\begin{array}{cc} 
\pm \frac{\delta_{s}}{2}+\Delta_{s} & \pm K_{s} \\
\mp K_{s}^{*} & \mp \frac{\delta_{s}}{2}+\Delta_{s}
\end{array}\right] .
$$

The equations for the first-order terms $\hat{A}_{s_{F}}^{(1)}\left(z, \omega_{s}\right)$ and $\hat{A}_{s_{B}}^{(1)}\left(z, \omega_{s}\right)$ have a more complex structure:

$$
\begin{aligned}
\frac{d \hat{A}_{s_{F}}^{(1)}\left(z, \omega_{s}\right)}{d z}= & i \frac{\delta_{s}\left(\omega_{s}\right)}{2} \hat{A}_{s_{F}}^{(1)}\left(z, \omega_{s}\right)+i K_{s}\left(\omega_{s}\right) \hat{A}_{s_{B}}^{(1)}\left(z, \omega_{s}\right) \\
+4 \int_{0}^{\infty} & d \omega_{s}^{\prime} K_{n l, q}\left(\omega_{s}, \omega_{s}^{\prime}\right) \exp \left[i \delta_{n l, q} z\right] \\
& \times \hat{A}_{p_{F}}\left(z, \omega_{s}+\omega_{s}^{\prime}\right) \hat{A}_{s_{F}}^{(0) \dagger}\left(z, \omega_{s}^{\prime}\right), \\
\frac{d \hat{A}_{s_{B}}^{(1)}\left(z, \omega_{s}\right)}{d z}= & -i \frac{\delta_{s}\left(\omega_{s}\right)}{2} \hat{A}_{s_{B}}^{(1)}\left(z, \omega_{s}\right)-i K_{s}^{*}\left(\omega_{s}\right) \hat{A}_{s_{F}}^{(1)}\left(z, \omega_{s}\right) \\
-4 \int_{0}^{\infty} & d \omega_{s}^{\prime} K_{n l, q}\left(\omega_{s}, \omega_{s}^{\prime}\right) \exp \left[-i \delta_{n l, q} z\right] \\
& \times \hat{A}_{p_{B}}\left(z, \omega_{s}+\omega_{s}^{\prime}\right) \hat{A}_{s_{B}}^{(0) \dagger}\left(z, \omega_{s}^{\prime}\right) .
\end{aligned}
$$

Equations (18) for a fixed frequency $\omega_{s}$ represent a coupled set of two linear differential equations with nonzero right-hand sides. They can be formally written as follows

$$
\frac{d A(z)}{d z}=i K A(z)+F(z),
$$

where $A$ and $F$ are vectors with 2 elements and $K$ is a $2 \times$ 2 matrix. Direct inspection confirms a non-homogeneous solution to Eq. (19) in the form

$$
A(z)=\int_{0}^{z} d z^{\prime} A^{\mathrm{hom}}\left(z-z^{\prime}\right) F\left(z^{\prime}\right)
$$

provided that $d A^{\mathrm{hom}}(z) /(d z)=i K A^{\mathrm{hom}}(z)$.

The sum of the zeroth- and first-order solutions gives us the solution to the equations (8) valid up to $K_{n l, q}$.
It can be again written in the matrix form using the matrices $\tilde{B}_{s}^{ \pm}\left(\omega_{s}\right)$ from Eq. (17):

$$
\begin{aligned}
& {\left[\begin{array}{l}
\hat{A}_{s_{F}}\left(z, \omega_{s}\right) \\
\hat{A}_{s_{B}}\left(z, \omega_{s}\right)
\end{array}\right] }=\sum_{ \pm} \tilde{B}_{s}^{ \pm}\left(\omega_{s}\right) \exp \left[ \pm i \Delta_{a}\left(\omega_{a}\right) z\right] \\
& \times\left[\begin{array}{c}
\hat{A}_{s_{F}}\left(0, \omega_{s}\right) \\
\hat{A}_{s_{B}}\left(0, \omega_{s}\right)
\end{array}\right]+\left[\begin{array}{l}
\hat{F}_{s_{F}}\left(z, \omega_{s}\right) \\
\hat{F}_{s_{B}}\left(z, \omega_{s}\right)
\end{array}\right] .
\end{aligned}
$$

Operators $\hat{F}_{s_{b}}\left(z, \omega_{s}\right)$ for $b=F, B$ describe the solution proportional to the first power of nonlinear constants $K_{n l, q}$. They are expressed for $z=L$ below in Eq. (23).

Now we write the solution in Eq. (21) for $z=L$ and partially invert the obtained linear relations in order to find the input-output relations of the waveguide. The result can be expressed as

$$
\begin{aligned}
& {\left[\begin{array}{c}
\hat{A}_{s_{F}}\left(L, \omega_{s}\right) \\
\hat{A}_{s_{B}}\left(0, \omega_{s}\right)
\end{array}\right]=} \\
& \quad D_{s}\left[\begin{array}{cc}
2 \Delta_{s} & 2 i K_{s} \sin \left(\Delta_{s} L\right) \\
2 i K_{s}^{*} \sin \left(\Delta_{s} L\right) & 2 \Delta_{s}
\end{array}\right]\left[\begin{array}{c}
\hat{A}_{s_{F}}\left(0, \omega_{s}\right) \\
\hat{A}_{s_{B}}\left(L, \omega_{s}\right)
\end{array}\right] \\
& \quad+\left[\begin{array}{c}
\hat{F}_{s_{F}}\left(L, \omega_{s}\right)-2 i K_{s} D_{s} \sin \left(\Delta_{s} L\right) \hat{F}_{s_{B}}\left(L, \omega_{s}\right) \\
-2 \Delta_{s} D_{s} \hat{F}_{s_{B}}\left(L, \omega_{s}\right)
\end{array}\right] .(22)
\end{aligned}
$$

In Eq. (22), the definition $D_{s}\left(\omega_{s}\right)=\left[2 \Delta_{s} \cos \left(\Delta_{s} L\right)-\right.$ $\left.i \delta_{s} \sin \left(\Delta_{s} L\right)\right]^{-1}$ has been used. The expressions on the right-hand side of Eq. (22) can be substantially simplified if the signal field fulfils the resonance condition $\Delta_{s} \approx m \pi / L, m=1,2, \ldots$, that gives the maximum enhancement of its electric-field amplitudes in the waveguide. In this case, $\sin \left(\Delta_{s} L\right) \approx 0$.

The operators $\hat{F}_{s_{b}}\left(L, \omega_{s}\right)$ at $z=L$ are obtained in the form:

$$
\begin{aligned}
& \hat{F}_{s_{a}}\left(L, \omega_{s}\right)=( \pm 1)_{a} 4 \int_{0}^{\infty} d \omega_{s}^{\prime} K_{n l, q}\left(\omega_{s}, \omega_{s}^{\prime}\right) \\
& \times \exp \left[( \pm 1)_{a} \frac{i \delta_{n l, q} L}{2}\right] \sum_{ \pm_{s}} \sum_{b=F, B} \tilde{B}_{s, a b}^{ \pm_{s}} \exp \left[ \pm_{s} \frac{i \Delta_{s}\left(\omega_{s}\right) L}{2}\right] \\
& \times \sum_{ \pm_{p}} \sum_{c=F, B} B_{p, b c}^{ \pm_{p}} \exp \left[ \pm_{p} \frac{i \Delta_{p}\left(\omega_{s}+\omega_{s}^{\prime}\right) L}{2}\right] \hat{A}_{p c}\left(\omega_{s}+\omega_{s}^{\prime}\right) \\
& \times \sum_{d=F, B} B_{s, b d}^{ \pm_{s} *} \exp \left[\mp s \frac{i \Delta_{s}\left(\omega_{s}^{\prime}\right) L}{2}\right] \hat{A}_{s d}^{\dagger}\left(\omega_{s}^{\prime}\right) S_{a}^{ \pm_{p}, \mp s}\left(\omega_{s}, \omega_{s}^{\prime}\right), \\
& a=F, B,
\end{aligned}
$$

where

$$
\begin{gathered}
S_{a}^{ \pm_{p}, \pm_{s}}\left(\omega_{s}, \omega_{s}^{\prime}\right)=2 L \operatorname{sinc}\left[\left[( \pm 1)_{a} \delta_{n l, q} \pm_{p} \Delta_{p}\left(\omega_{s}+\omega_{s}^{\prime}\right)\right.\right. \\
\left.\left. \pm_{s} \Delta_{s}\left(\omega_{s}\right) \pm_{s} \Delta_{s}\left(\omega_{s}^{\prime}\right)\right] L / 2\right], \quad a=F, B,
\end{gathered}
$$

$\operatorname{sinc}(x)=\sin (x) / x$. The matrices $B_{s}^{ \pm}\left(\omega_{s}\right)$ introduced in Eq. (23) are defined analogously to those given in Eq. (13) and characterizing the fundamental field. We note that the expression in Eq. (23) contains only one half of all possible terms. The missing terms are far from the quasi-phase-matching conditions and thus give negligible contributions. 


\section{B. Gaussian spectral approximation in a resonant term}

The right-hand-side of Eq. (23) giving $\hat{F}_{s_{a}}\left(L, \omega_{s}\right)$ is composed of four terms that differ in the signs of eigenvalues $\Delta_{p}$ and $\Delta_{s}$ (resolved by the symbols $\pm_{p}, \pm_{s}$ ). Quasi-phase-matching conditions in the waveguide are such that they emphasize only one out of the four terms. The remaining terms give small contributions. That is why we pay attention only to one of them. We also consider only the incident forward-propagating fundamental field in a multi-mode coherent state with a Gaussian spectral shape and spectral phase variations such that the fundamental field in the middle of the waveguide has the same phase along the spectrum:

$$
\begin{aligned}
A_{p_{F}}\left(0, \omega_{p}\right)= & \xi_{p} \sqrt{\frac{\tau_{p}}{\sqrt{2 \pi}^{3}}} \exp \left[-\frac{\tau_{p}^{2}\left(\omega_{p}-\omega_{p}^{0}\right)^{2}}{4}\right] \\
& \times \exp \left[\mp_{p} \frac{i \Delta_{p}\left(\omega_{p}\right) L}{2}\right], \\
A_{p_{B}}\left(L, \omega_{p}\right)= & 0 .
\end{aligned}
$$

The fundamental pulse has amplitude $\xi_{p}$, duration $\tau_{p}$ and carrying frequency $\omega_{p}^{0}$.

We further assume that both the fundamental and SSF spectra are not too wide and so the propagation constants $\beta_{a}\left(\omega_{a}\right)$ can be approximated by their second-order Taylor expansions:

$$
\begin{aligned}
\beta_{a}\left(\omega_{a}\right) & =\beta_{a}^{0}+\beta_{1 a}\left(\omega_{a}-\omega_{a}^{0}\right)+\beta_{2 a}\left(\omega_{a}-\omega_{a}^{0}\right)^{2}, \\
\beta_{a}^{0} & =\beta_{a}\left(\omega_{a}^{0}\right), \\
\beta_{i a} & =\left.\frac{1}{i !} \frac{d^{i} \beta_{a}}{d \omega_{a}^{i}}\right|_{\omega_{a}=\omega_{a}^{0}}, \quad i=1,2 ; \quad a=p, s .
\end{aligned}
$$

In this approximation, the eigenvalues $\Delta_{a}\left(\omega_{a}\right)$ can be expressed as

$$
\begin{aligned}
\Delta_{a}\left(\omega_{a}\right) & =\Delta_{a}^{0}+\Delta_{1 a}\left(\omega_{a}-\omega_{a}^{0}\right)+\Delta_{2 a}\left(\omega_{a}-\omega_{a}^{0}\right)^{2}, \\
\Delta_{a}^{0} & =\Delta_{a}\left(\omega_{a}^{0}\right), \\
\Delta_{1 a}= & \frac{\beta_{1 a}\left(\beta_{a}^{0}-\pi / \Lambda_{a}\right)}{\Delta_{a}^{0}}, \\
\Delta_{2 a}= & \frac{\beta_{2 a}\left(\beta_{a}^{0}-\pi / \Lambda_{a}\right)+\beta_{1 a}^{2}}{2 \Delta_{a}^{0}}-\frac{\beta_{1 a}^{2}\left(\beta_{a}^{0}-\pi / \Lambda_{a}\right)^{2}}{2\left(\Delta_{a}^{0}\right)^{3}}, \\
& a=p, s .
\end{aligned}
$$

Efficient nonlinear interaction occurs if quasi-phasematching conditions for the central frequencies $\omega_{p}^{0}$ and $\omega_{s}^{0}=\omega_{p}^{0} / 2$ are fulfilled, i.e.

$$
\delta_{n l, q} \pm_{p} \Delta_{p}\left(\omega_{p}^{0}\right) \mp_{s} 2 \Delta_{s}\left(\omega_{s}^{0}\right)=0 .
$$

The function $S_{a}^{ \pm_{p}, \pm_{s}}$ in Eq. (24) can then be rewritten as

$$
\begin{gathered}
S^{ \pm_{p}, \pm_{s}}\left(\omega_{s}, \omega_{s}^{\prime}\right)=2 L \operatorname{sinc}\left(\left[\left( \pm_{p} \Delta_{1 p} \pm_{s} \Delta_{1 s}\right)\right.\right. \\
\left.\left.\times\left(\omega_{s}+\omega_{s}^{\prime}-\omega_{p}^{0}\right) \pm_{s} \frac{\Delta_{2 s}\left(\omega_{s}-\omega_{s}^{\prime}\right)^{2}}{2}\right] \frac{L}{2}\right)
\end{gathered}
$$

assuming $\Delta_{1 a} \gg \Delta_{2 a} \Delta \omega$ for $a=p, s ; \Delta \omega$ characterizes the fields' spectral width.

The use of Gaussian approximation to the sinc function in Eq. (29) $\left[\operatorname{sinc}\left(\alpha x+\beta y^{2}\right) \approx \exp \left(-\alpha^{2} x^{2} / 5-|\beta| y^{2} / 3\right)\right]$ for constants $\alpha, \beta]$ allows to derive the useful relation:

$$
\begin{gathered}
S^{ \pm_{p}, \pm_{s}}\left(\omega_{s}, \omega_{s}^{\prime}\right) A_{p_{F}}\left(0, \omega_{s}+\omega_{s}^{\prime}\right)=2 L \xi_{p} \sqrt{\frac{\tau_{p}}{\sqrt{2 \pi}^{3}}} \\
\quad \times \exp \left[\mp p \frac{i \Delta_{p}\left(\omega_{s}+\omega_{s}^{\prime}\right) L}{2}\right] \Phi\left(\omega_{s}, \omega_{s}^{\prime}\right) .
\end{gathered}
$$

In Eq. (30), the function $\Phi$,

$$
\begin{aligned}
\Phi\left(\omega_{s}, \omega_{s}^{\prime}\right) & =\exp \left[-\frac{\left(\omega_{s}-\omega_{s}^{\prime}\right)^{2}}{\Delta_{-}^{2}}-\frac{\left(\omega_{s}+\omega_{s}^{\prime}-\omega_{p}^{0}\right)^{2}}{\Delta_{+}^{2}}\right] \\
\frac{1}{\Delta_{-}^{2}} & =\frac{\Delta_{2 s} L}{12} \\
\frac{1}{\Delta_{+}^{2}} & =\frac{\left( \pm_{p} \Delta_{1 p} \pm_{s} \Delta_{1 s}\right)^{2} L^{2}}{20}+\frac{\tau_{p}^{2}}{4}
\end{aligned}
$$

determines the structure of spectral modes.

These spectral modes can be revealed using the Schmidt decomposition [49, 61] of function $\Phi$ :

$$
\Phi\left(\omega_{s}, \omega_{s}^{\prime}\right)=\sum_{n=0}^{\infty} \mu_{n} \phi_{n}\left(\omega_{s}\right) \phi_{n}\left(\omega_{s}^{\prime}\right) .
$$

Eigenvalues $\mu_{n}$ introduced in Eq. (33) take the form:

$$
\begin{aligned}
\mu_{n} & =2 \sqrt{\frac{\pi}{\Delta_{+} \Delta_{-}}} \theta^{n / 2}, \\
\theta & =\left(\frac{\Delta_{+}-\Delta_{-}}{\Delta_{+}+\Delta_{-}}\right)^{2} .
\end{aligned}
$$

Eigenmode spectral functions $\phi_{n}$ can be expressed in terms of the Hermite polynomials $\mathrm{H}_{\mathrm{n}}$ :

$$
\begin{aligned}
\phi_{n}\left(\omega_{s}\right)= & \sqrt{\frac{\tau_{s}}{2^{n} n ! \sqrt{\pi}}} \exp \left[-\tau_{s}^{2}\left(\omega_{s}-\omega_{s}^{0}\right)^{2}\right] \\
& \times \mathrm{H}_{n}\left(\tau_{s}\left[\omega_{s}-\omega_{s}^{0}\right]\right), \\
\tau_{s}= & \sqrt{\frac{1-\theta^{2}}{\theta}} .
\end{aligned}
$$

For the considered waveguide, $\Delta_{+} \ll \Delta_{-}$and thus a typical time constant $\tau_{s}$ of the SSF field defined in Eq. (36) can be approximated as:

$$
\tau_{s} \approx 8 \frac{\Delta_{+}}{\Delta_{-}} .
$$

The Schmidt decomposition in Eq. (33) allows us to transform Eqs. (22) written for the 'continuous index' $\omega_{s}$ to those related to spectral modes. The appropriate 
unitary transformation of the input and output operators takes the form:

$$
\begin{aligned}
\hat{a}_{s_{F}, n}^{\text {in }}= & \int_{0}^{\infty} \quad d \omega_{s} \phi_{n}\left(\omega_{s}\right) \hat{a}_{s_{F}}\left(0, \omega_{s}\right) \exp \left[-\frac{i \pi L}{2 \Lambda_{s}}\right] \\
& \times \exp \left[\mp_{s} \frac{i \Delta_{s}\left(\omega_{s}\right) L}{2}\right], \\
\hat{a}_{s_{B}, n}^{\text {in }}= & \int_{0}^{\infty} d \omega_{s} \phi_{n}\left(\omega_{s}\right) \hat{a}_{s_{B}}\left(L, \omega_{s}\right) \exp \left[\frac{i \pi L}{2 \Lambda_{s}}\right] \\
& \times \exp \left[ \pm_{s} \frac{i \Delta_{s}\left(\omega_{s}\right) L}{2}\right], \\
\hat{a}_{s_{F}, n}^{\text {out }}= & \int_{0}^{\infty} d \omega_{s} \phi_{n}\left(\omega_{s}\right) \hat{a}_{s_{F}}\left(L, \omega_{s}\right) \exp \left[-\frac{i \pi L}{2 \Lambda_{s}}\right] \\
& \times \exp \left[\mp_{s} \frac{i \Delta_{s}\left(\omega_{s}\right) L}{2}\right], \\
\hat{a}_{s_{B}, n}^{\text {out }}= & \int_{0}^{\infty} d \omega_{s} \phi_{n}\left(\omega_{s}\right) \hat{a}_{s_{B}}\left(0, \omega_{s}\right) \exp \left[\frac{i \pi L}{2 \Lambda_{s}}\right] \\
& \times \exp \left[ \pm_{s} \frac{i \Delta_{s}\left(\omega_{s}\right) L}{2}\right] .
\end{aligned}
$$

When weak spectral dependencies of multiplicative factors occurring in Eqs. (22) and (23) are neglected, the transformed Eqs. (22) valid either close to the resonance condition or for non-scattered SSF field are expressed as:

$$
\begin{aligned}
{\left[\begin{array}{c}
\hat{a}_{s_{F}, n}^{\text {out }} \\
\hat{a}_{s_{B}, n}^{\text {out }}
\end{array}\right]=} & 2 \Delta_{s}^{0} D_{s}^{0} \exp \left[\frac{i \pi L}{\Lambda_{s}}\right]\left[\begin{array}{ll}
1 & 0 \\
0 & 1
\end{array}\right]\left[\begin{array}{c}
\hat{a}_{s_{F}, n}^{\text {in }} \\
\hat{a}_{s_{B}, n}^{\text {in }}
\end{array}\right] \\
& +\left[\begin{array}{c}
\hat{F}_{s_{F}, n} \\
\hat{F}_{s_{B}, n}
\end{array}\right]
\end{aligned}
$$

$D_{s}^{0}=D_{s}\left(\omega_{s}^{0}\right)$

Defining suitable nonlinear coupling constants $\xi_{n}$ for individual spectral modes,

$$
\xi_{n}=8 L K_{n l, q}\left(\omega_{s}^{0}, \omega_{s}^{0}\right) \sqrt{\frac{\tau_{p}}{\sqrt{2 \pi}^{3}}} \xi_{p} \mu_{n},
$$

the operator coefficients $\hat{F}_{s_{F}, n}$ and $\hat{F}_{s_{B}, n}$ introduced in Eq. (39) take the form:

$$
\begin{aligned}
& \hat{F}_{s_{F}, n}=\xi_{n} \exp \left[\frac{i \delta_{n l, q} L}{2}\right] \frac{D_{p}^{0} D_{s}^{0 *}}{2 \Delta_{s}^{0}}\left\{\left[\left( \pm_{s} \frac{\delta_{s}^{0}}{2}+\Delta_{s}^{0}\right)^{2}\right.\right. \\
& \left.\times\left( \pm_{p} \frac{\delta_{p}^{0}}{2}+\Delta_{p}^{0}\right) \pm_{p} K_{p}^{0 *}\left(K_{s}^{0}\right)^{2}\right] \hat{a}_{s_{F}, n}^{\mathrm{in} \dagger}+\left[\left(\frac{\delta_{s}^{0}}{2} \pm_{s} \Delta_{s}^{0}\right)\right. \\
& \left.\left.\times K_{s}^{0 *}\left( \pm_{p} \frac{\delta_{p}^{0}}{2}+\Delta_{p}^{0}\right) \pm_{p} K_{s}^{0} K_{p}^{0 *}\left(\frac{\delta_{s}^{0}}{2} \mp_{s} \Delta_{s}^{0}\right)\right] \hat{a}_{s_{B}, n}^{\mathrm{in} \dagger}\right\} \\
& \hat{F}_{s_{B}, n}=-\xi_{n} \exp \left[-\frac{i \delta_{n l, q} L}{2}\right] \exp \left( \pm_{s} i \Delta_{s}^{0} L\right) D_{p}^{0}\left|D_{s}^{0}\right|^{2} \\
& \times\left\{\left[K_{s}^{0 *}\left(\frac{\delta_{s}^{0}}{2} \pm_{s} \Delta_{s}^{0}\right)\left(\mp p \frac{\delta_{p}^{0}}{2}-\Delta_{p}^{0}\right) \mp_{p} K_{p}^{0 *} K_{s}^{0 *}\right.\right. \\
& \left.\times\left(\frac{\delta_{s}^{0}}{2} \mp_{s} \Delta_{s}^{0}\right)\right] \hat{a}_{s_{F}, n}^{\mathrm{in} \dagger}+\left[\left(\mp_{p} \frac{\delta_{p}^{0}}{2}-\Delta_{p}^{0}\right)\left(K_{s}^{0 *}\right)^{2}\right.
\end{aligned}
$$

$$
\left.\left.\mp_{p} K_{p}^{0 *}\left(\frac{\delta_{s}^{0}}{2} \mp_{s} \Delta_{s}^{0}\right)^{2}\right] \hat{a}_{s_{B}, n}^{\mathrm{in} \dagger}\right\}
$$

$D_{p}^{0}=D_{p}\left(\omega_{p}^{0}\right)$.

If the corrugation is only in the fundamental field, the expressions for operator coefficients $\hat{F}_{s_{F}, n}$ and $\hat{F}_{s_{B}, n}$ in Eq. (41) simplify:

$$
\begin{aligned}
\hat{F}_{s_{F}, n}= & \xi_{n} \exp \left[\frac{i \delta_{n l, q} L}{2}\right] D_{p}^{0} \exp \left(-i \beta_{s}^{0} L\right) \\
& \times\left( \pm_{p} \frac{\delta_{p}^{0}}{2}+\Delta_{p}^{0}\right) \hat{a}_{s_{F}, n}^{\mathrm{in} \dagger}, \\
\hat{F}_{s_{B}, n}= & -\xi_{n} \exp \left[\frac{-i \delta_{n l, q} L}{2}\right] D_{p}^{0} \exp \left(-i \beta_{s}^{0} L\right) \\
& \times\left(\mp_{p} K_{p}^{0 *}\right) \hat{a}_{s_{B}, n}^{\mathrm{in \dagger}} .
\end{aligned}
$$

On the other hand, the corrugation resonating with the SSF field leads to the following expressions

$$
\begin{gathered}
\hat{F}_{s_{F}, n}=\xi_{n} \exp \left[\frac{i \delta_{n l, q} L}{2}\right] \exp \left(i \beta_{p}^{0} L\right) \frac{D_{s}^{0 *}}{2 \Delta_{s}^{0}}\left( \pm_{s} \frac{\delta_{s}^{0}}{2}+\Delta_{s}^{0}\right) \\
\times\left\{\left( \pm_{s} \frac{\delta_{s}^{0}}{2}+\Delta_{s}^{0}\right) \hat{a}_{s_{F}, n}^{\mathrm{in} \dagger} \pm K_{s}^{0 *} \hat{a}_{s_{B}, n}^{\mathrm{in} \dagger}\right\}, \\
\hat{F}_{s_{B}, n}=-\xi_{n} \exp \left[-\frac{i \delta_{n l, q} L}{2}\right] \exp \left(i \beta_{p}^{0} L\right) \exp \left( \pm_{s} i \Delta_{s}^{0} L\right) \\
\times\left|D_{s}^{0}\right|^{2} K_{s}^{0 *}\left\{\left(-\frac{\delta_{s}^{0}}{2} \mp_{s} \Delta_{s}^{0}\right) \hat{a}_{s_{F}, n}^{\mathrm{in} \dagger}-K_{s}^{0 *} \hat{a}_{s_{B}, n}^{\mathrm{in} \dagger}\right\} .
\end{gathered}
$$

Multiplicative factors occurring in the expressions in Eqs. (41- 43) describe the enhancement of nonlinear interaction due to scattering of the fundamental and SSF fields. This enhancement can be quantified by the expression $\left( \pm_{a} \delta_{a}^{0} / 2+\Delta_{a}^{0}\right) /\left(2 \Delta_{a}^{0}\right)$ for $a=p, s$. Let us consider first the corrugation present in the fundamental field. In this case, the quasi-phase-matching condition written in Eq. (28) takes the form

$$
\delta_{n l, q}^{\text {nat }, 0}-\frac{\delta_{p}^{0}}{2} \pm_{p} \Delta_{p}^{0}=0
$$

where $\delta_{n l, q}^{\text {nat }, 0}=\beta_{p}^{0}-2 \beta_{s}^{0}+2 \pi q / \Lambda_{n l}$ describes the natural quasi-phase mismatch. Efficient quasi-phase-matching can be reached only if $\delta_{n l, q}^{\text {nat, }}$ and $\delta_{p}^{0}$ have the same sign [28]. Considering a positive value of phase mismatch $\delta_{n l, q}^{\text {nat, }}$, the sign $+[-]$ in Eq. (44) is suitable for $\delta_{n l, q}^{\text {nat, }}<\left|K_{p}^{0}\right|\left[\delta_{n l, q}^{\text {nat, }}>\left|K_{p}^{0}\right|\right]$. The enhancement of fundamental-field amplitudes is then described by the expression

$$
\frac{\delta_{p}^{0} / 2+\Delta_{p}^{0}}{2 \Delta_{p}^{0}}=\frac{1 \pm_{p} 1}{2}+\frac{\delta_{n l, q}^{\text {nat }, 0}}{2 \Delta_{p}^{0}}>1
$$

On the other hand, a negative value of phase mismatch $\delta_{n l, q}^{\text {nat, } 0}$ requires the opposite choice of signs in Eq. (44) and 
the enhancement of fundamental-field amplitudes can be quantified by the expression

$$
\frac{-\delta_{p}^{0} / 2+\Delta_{p}^{0}}{2 \Delta_{p}^{0}}=\frac{1 \mp_{p} 1}{2}-\frac{\delta_{n l, q}^{\text {nat }, 0}}{2 \Delta_{p}^{0}}>1 .
$$

The presence of corrugation in the SSF field needs the following quasi-phase-matching conditions:

$$
\delta_{n l, q}^{\text {nat }, 0}+\delta_{s}^{0} \mp s \Delta_{s}^{0}=0 .
$$

In this case, the quantities $\delta_{n l, q}^{\text {nat, } 0}$ and $\delta_{s}^{0}$ have to differ in their signs. The enhancement factors of the SSF-field amplitudes can be analyzed similarly as for the fundamental field.

\section{Principal squeeze variance}

The enhancement of electric-field amplitudes due to the presence of the corrugation results in larger squeezing of fluctuations of these amplitudes. The suppression of amplitude fluctuations can be quantified by a principal squeeze variance $\lambda[2,3,23]$ that can be determined along the relations

$$
\begin{aligned}
\lambda_{s_{b}} & =1+2\left(B_{s_{b}}-\left|C_{s_{b}}\right|\right), \\
B_{s_{b}} & =\left\langle\Delta \hat{a}_{s_{b}}^{\dagger} \Delta \hat{a}_{s_{b}}\right\rangle, \\
C_{s_{b}} & =\left\langle\left(\Delta \hat{a}_{s_{b}}\right)^{2}\right\rangle, \quad b=F, B .
\end{aligned}
$$

In Eqs. (49), $\Delta \hat{a}=\hat{a}-\langle\hat{a}\rangle$ and symbol \langle\rangle means the quantum mechanical mean value.

Substitution of the expressions in Eq. (41) into Eqs. (48) and (49) provides the formulas for principal squeeze variances $\lambda_{s_{F}, n}$ and $\lambda_{s_{B}, n}$ of individual spectral modes,

$$
\begin{aligned}
& \lambda_{s_{F}, n}=1-\frac{\left|\xi_{n}\right|}{4 \Delta_{p}^{0}\left(\Delta_{s}^{0}\right)^{2}} \mid\left( \pm_{s} \frac{\delta_{s}^{0}}{2}+\Delta_{s}^{0}\right)\left( \pm_{p} \frac{\delta_{p}^{0}}{2}+\Delta_{p}^{0}\right) \\
& \times\left( \pm_{s} \frac{\delta_{s}^{0}}{2}+\Delta_{s}^{0} \pm K_{s}^{0 *}\right) \pm_{p} K_{p}^{0 *} K_{s}^{0}\left(\frac{\delta_{s}^{0}}{2} \mp_{s} \Delta_{s}^{0}+K_{s}^{0}\right) \mid \\
& \lambda_{s_{B}, n}=1-\frac{\left|\xi_{n}\right|}{4 \Delta_{p}^{0}\left(\Delta_{s}^{0}\right)^{2}} \mid K_{s}^{0 *}\left( \pm_{p} \frac{\delta_{p}^{0}}{2}+\Delta_{p}^{0}\right) \\
& \times\left(-\frac{\delta_{s}^{0}}{2} \mp_{s} \Delta_{s}^{0}-K_{s}^{0 *}\right) \mp_{p} K_{p}^{0 *}\left(\mp s_{s} \frac{\delta_{s}^{0}}{2}+\Delta_{s}^{0}\right) \\
& \times\left(\mp s \frac{\delta_{s}^{0}}{2}+\Delta_{s}^{0} \mp{ }_{s} K_{s}^{0}\right) \mid .
\end{aligned}
$$

The enhancement factors discussed for the fundamental field in Eqs. (45) and (46) are clearly recognized in the expressions (50). Also the factors $\delta_{s}^{0} / 2 \pm_{s} \Delta_{s}^{0}+K_{s}^{0}$ and $\delta_{s}^{0} / 2 \pm_{s} \Delta_{s}^{0}+K_{s}^{0 *}$ found in Eqs. (50) significantly contribute to the enhancement of squeezing of SSF electricfield amplitudes due to the same signs in front of $\delta_{s}^{0}$ and $K_{s}^{0}$.
The formulas in Eq. (50) considerably simplify for the corrugation in the fundamental field only:

$$
\begin{aligned}
& \lambda_{s_{F}, n}=1-\frac{\left|\xi_{n}\right|}{\Delta_{p}^{0}}\left|\left( \pm_{p} \frac{\delta_{p}^{0}}{2}+\Delta_{p}^{0}\right)\right|, \\
& \lambda_{s_{B}, n}=1-\frac{\left|\xi_{n} K_{p}^{0}\right|}{\Delta_{p}^{0}} .
\end{aligned}
$$

On the other hand, the presence of corrugation only in the SSF field leaves us with the expressions

$$
\begin{aligned}
\lambda_{s_{F}, n}=1 & -\frac{\left|\xi_{n}\right|}{2\left(\Delta_{s}^{0}\right)^{2}} \mid\left( \pm_{s} \frac{\delta_{s}^{0}}{2}+\Delta_{s}^{0}\right) \\
& \times\left( \pm_{s} \frac{\delta_{s}^{0}}{2}+\Delta_{s}^{0} \pm K_{s}^{0 *}\right) \mid \\
\lambda_{s_{B}, n}=1 & -\frac{\left|\xi_{n} K_{s}^{0}\right|}{2\left(\Delta_{s}^{0}\right)^{2}}\left|\left( \pm_{s} \frac{\delta_{s}^{0}}{2}+\Delta_{s}^{0} \pm_{s} K_{s}^{0 *}\right)\right| .
\end{aligned}
$$

\section{NON-PERTURBATION SOLUTION FOR NON-DISPERSION PROPAGATION}

The linear coupling constants $K_{p}, K_{s}$ and nonlinear coupling constants $K_{n l, q}$ depend usually only weakly on frequencies in a relatively wide interval. Weak frequency dependence of the coupling constants $K_{p}, K_{s}$ and $K_{n l, 1}$ in the considered range [approx. $80 \mathrm{~nm}(40 \mathrm{~nm})$ for the fundamental (SSF) field] is shown in Figs. 2a, 3a, and 4 a, respectively, for the analyzed waveguide. Also inter-mode dispersion both in the fundamental and SSF fields can be in the first approximation neglected (see Figs. 2 b, 3b, and 4b for frequency dependence of the linear phase mismatches $\delta_{p}$ and $\delta_{s}$ and nonlinear phase mismatch $\left.\delta_{n l, 1}\right)$. We note that the needed spectral range of the SSF field roughly depends inversely proportionally on the waveguide length $L$. The solution of operator equations considerably simplifies when the frequency dependencies are omitted and even certain analytical results can be obtained.

In this case, Eqs. (8) and (9) for the operator amplitudes $\hat{A}_{a_{b}}(z, \omega), a=p, s, b=F, B$ can be decoupled in their frequency 'index' using the Fourier transform:

$$
\begin{aligned}
& \hat{A}_{a_{b}}\left(z, \tau_{a}\right)=\int_{-\infty}^{\infty} d \omega_{a} \hat{A}_{a_{b}}\left(z, \omega_{a}\right) \exp \left(-i \omega_{a} \tau_{a}\right) \\
& \hat{A}_{a_{b}}\left(z, \omega_{a}\right)=\frac{1}{2 \pi} \int_{-\infty}^{\infty} d \tau_{a} \hat{A}_{a_{b}}\left(z, \tau_{a}\right) \exp \left(i \omega_{a} \tau_{a}\right)
\end{aligned}
$$

After the transformation, Eqs. (8) attain the form:

$$
\begin{gathered}
\frac{d \hat{A}_{s_{F}}(z, \tau)}{d z}=i \frac{\delta_{s}^{0}}{2} \hat{A}_{s_{F}}(z, \tau)+i K_{s}^{0} \hat{A}_{s_{B}}(z, \tau) \\
\quad+4 K_{n l, q}^{0} \exp \left[i \delta_{n l, q} z\right] \hat{A}_{p_{F}}(z, \tau) \hat{A}_{s_{F}}^{\dagger}(z, \tau) \\
\frac{d \hat{A}_{s_{B}}(z, \tau)}{d z}=-i \frac{\delta_{s}^{0}}{2} \hat{A}_{s_{B}}(z, \tau)-i K_{s}^{0 *} \hat{A}_{s_{F}}(z, \tau) \\
\quad-4 K_{n l, q}^{0} \exp \left[-i \delta_{n l, q} z\right] \hat{A}_{p_{B}}(z, \tau) \hat{A}_{s_{B}}^{\dagger}(z, \tau)
\end{gathered}
$$


a)

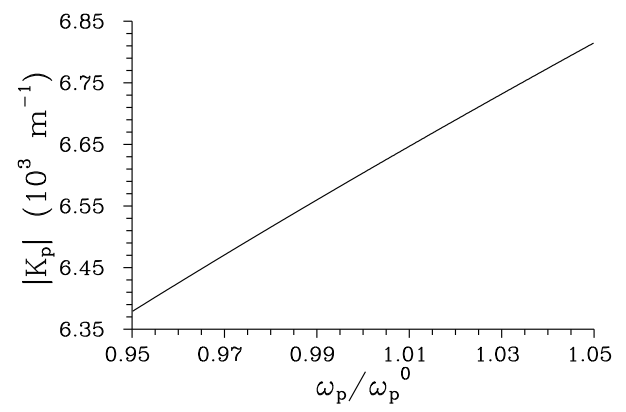

b)

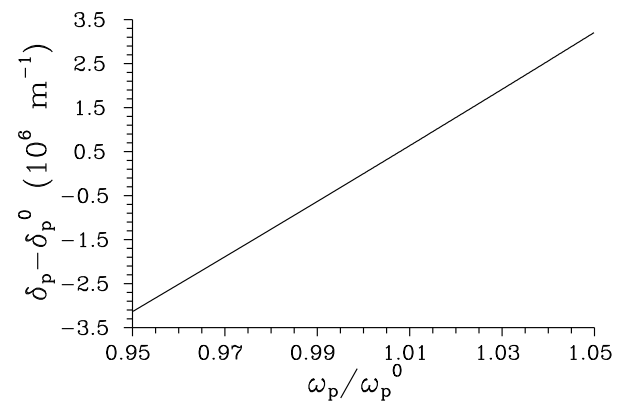

FIG. 2: (a) Absolute value of linear coupling constant $K_{p}$ and (b) linear phase mismatch $\delta_{p}-\delta_{p}^{0}$ for the fundamental field as they depend on relative frequency $\omega_{p} / \omega_{p}^{0} ; t=5 \times 10^{-7} \mathrm{~m}$, $t_{l}=5 \times 10^{-8} \mathrm{~m}$.

$K_{n l, q}^{0}=K_{n l, q}\left(\omega_{s}^{0}, \omega_{s}^{0}\right)$. The solution of Eqs. (9) written in Eq. (12) is transformed in the considered approximation of strong fundamental field as follows:

$$
\left[\begin{array}{c}
\hat{A}_{p_{F}}(z, \tau) \\
\hat{A}_{p_{B}}(z, \tau)
\end{array}\right]=\sum_{ \pm} B_{p}^{ \pm 0} \exp \left[ \pm i \Delta_{p}^{0} z\right]\left[\begin{array}{c}
\hat{A}_{p_{F}}(0, \tau) \\
\hat{A}_{p_{B}}(L, \tau)
\end{array}\right]
$$

and $B_{p}^{ \pm 0}=B_{p}^{ \pm}\left(\omega_{p}^{0}\right)$ defined in Eq. (13). The fundamental-field operator amplitudes $\hat{A}_{p_{F}}(0, \tau)$ and $\hat{A}_{p_{B}}(L, \tau)$ describe the incident pulses.

We further discuss the solution to Eqs. (55) for the corrugation present either in the fundamental or SSF field.

\section{A. Corrugation in the pump field only}

In this case, two operator equations (55) are independent. Moreover, we are interested in a solution close to the resonance where the nonlinear terms in Eqs. (55) give considerable contribution. We further pay attention to the SSF forward-propagating field and assume only the incident fundamental field at $z=0$. Using Eq. (56) the first equation in (55) can be rewritten for two different resonant conditions indicated by upper indices \pm ,

$$
\begin{aligned}
& \frac{d \hat{A}_{s_{F}}(z, \tau)}{d z}=i \frac{\delta_{s}^{0}}{2} \hat{A}_{s_{F}}(z, \tau)+4 K^{ \pm}(\tau) \\
& \quad \times \exp \left[i\left(\delta_{n l, q}^{0} \pm \Delta_{p}^{0}\right) z\right] \hat{A}_{s_{F}}^{\dagger}(z, \tau) .
\end{aligned}
$$

The effective nonlinear coupling constant $K^{ \pm}(\tau) \equiv$ $K_{n l, q}^{0} B_{p, F F}^{ \pm 0} A_{p_{F}}(0, \tau)$ incorporates the enhancement of a)

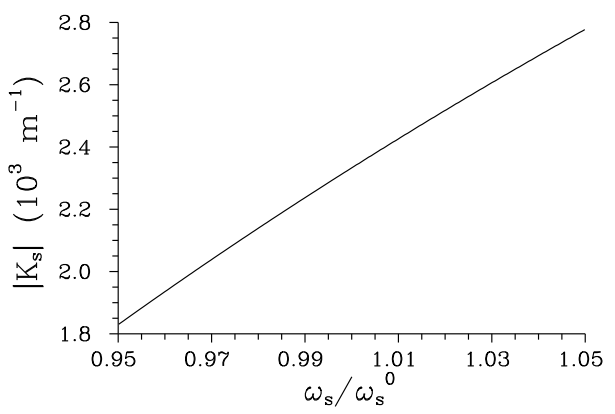

b)

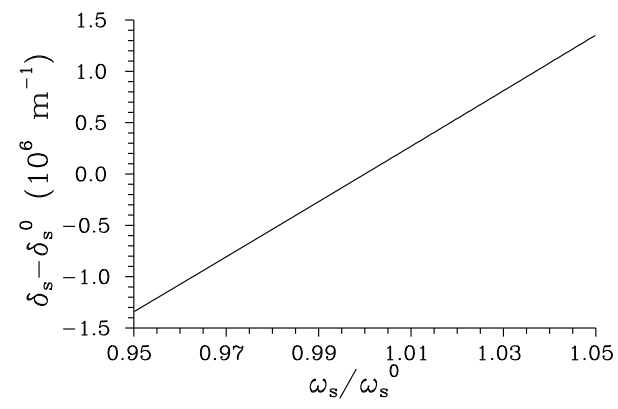

FIG. 3: (a) Absolute value of linear coupling constant $K_{s}$ and (b) linear phase mismatch $\delta_{s}-\delta_{s}^{0}$ for the SSF field as functions of relative frequency $\omega_{s} / \omega_{p}^{0}$; values of parameters are the same as in Fig. 2

a)

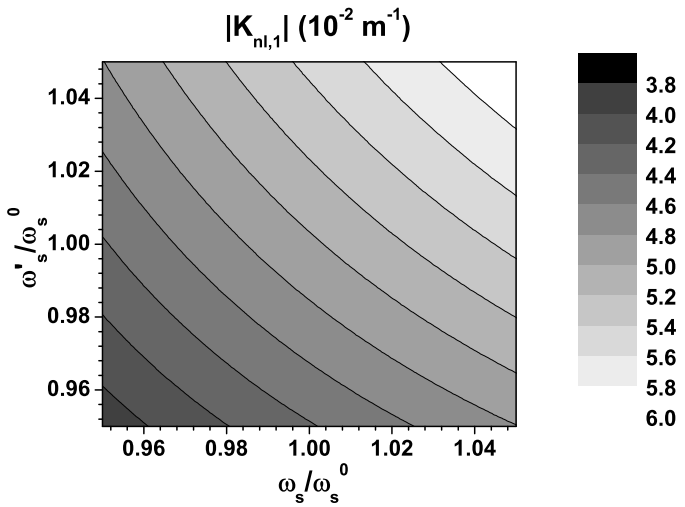

b)

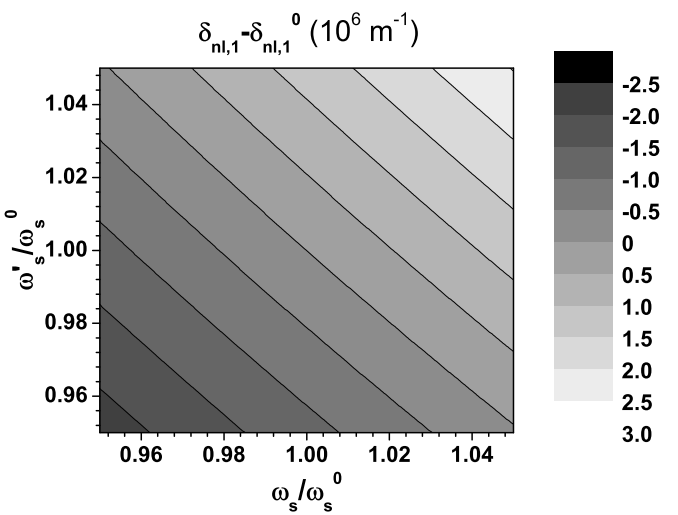

FIG. 4: Contour plots of (a) absolute value $\left|K_{n l, 1}\right|$ of nonlinear coupling constant and (b) nonlinear phase mismatch $\delta_{n l, 1}-\delta_{n l, 1}^{0}$ as they depend on relative frequencies $\omega_{s} / \omega_{p}^{0}$ and $\omega_{s}^{\prime} / \omega_{p}^{0}$. Values of parameters are the same as in Fig. 2. 
nonlinear interaction due to the fundamental-field scattering.

The substitution $\hat{A}_{s_{F}}(z, \tau)=\hat{\mathcal{A}}_{s_{F}}(z, \tau) \exp \left[i\left(\delta_{n l, q}^{0} \pm\right.\right.$ $\left.\left.\Delta_{p}^{0}\right) z / 2\right], \hat{A}_{s_{F}}^{\dagger}(z, \tau)=\hat{\mathcal{A}}_{S_{F}}^{\dagger}(z, \tau) \exp \left[-i\left(\delta_{n l, q}^{0} \pm \Delta_{p}^{0}\right) z / 2\right]$ in Eq. (57) leads to differential equations with constant coefficients obeyed by the operator amplitudes $\hat{\mathcal{A}}_{s_{F}}$ and $\hat{\mathcal{A}}_{S_{F}}^{\dagger}$. Their solution transformed to the original operators can be written as:

$$
\begin{aligned}
\hat{A}_{s_{F}}(L, \tau)= & U_{F F}(\tau) \hat{A}_{s_{F}}(0, \tau)+V_{F F}(\tau) \hat{A}_{s_{F}}^{\dagger}(0, \tau) \\
U_{F F}(\tau)= & \frac{1}{2} \exp \left(i \beta_{s}^{0} L\right) \exp \left(i \frac{\Omega^{ \pm} L}{2}\right) \\
& \times\left[\left(1-\frac{i \Omega^{ \pm}}{2 \lambda^{ \pm}(\tau)}\right) \exp \left[\lambda^{ \pm}(\tau) L\right]\right. \\
& \left.+\left(1+\frac{i \Omega^{ \pm}}{2 \lambda^{ \pm}(\tau)}\right) \exp \left[-\lambda^{ \pm}(\tau) L\right]\right] \\
V_{F F}(\tau)= & \frac{2 K^{ \pm}(\tau)}{\lambda^{ \pm}(\tau)} \exp \left(i \beta_{s}^{0} L\right) \exp \left(i \frac{\Omega^{ \pm} L}{2}\right) \\
& \times\left[\exp \left[\lambda^{ \pm}(\tau) L\right]-\exp \left[-\lambda^{ \pm}(\tau) L\right]\right]
\end{aligned}
$$

The phase mismatches $\Omega^{ \pm}$and eigenvalues $\lambda^{ \pm}$are given along the expressions:

$$
\begin{aligned}
\Omega^{ \pm} & =\delta_{n l, q}-\delta_{s}^{0} \pm \Delta_{p}^{0}, \\
\lambda^{ \pm}(\tau) & =\sqrt{16\left|K^{ \pm}(\tau)\right|^{2}-\Omega^{ \pm 2} / 4} .
\end{aligned}
$$

Ideal phase matching leads to $\Omega^{ \pm}=0$.

The inverse Fourier transform (54) of the expression for operator amplitude $\hat{A}_{s_{F}}(L, \tau)$ in Eq. (58) provides the spectral operator amplitude $\hat{A}_{s_{F}}\left(L, \omega_{s}\right)$ that equals the operator amplitude $\hat{a}_{s_{F}}^{\text {out }}\left(\omega_{s}\right)$ in the limit $\Lambda_{s} \longrightarrow \infty$,

$$
\begin{aligned}
\hat{a}_{s_{F}}^{\mathrm{out}}\left(\omega_{s}\right) & =\left[\int_{0}^{\infty} d \omega_{s}^{\prime} U_{F F}\left(\omega_{s}-\omega_{s}^{\prime}\right) \hat{a}_{s_{F}}^{\mathrm{in}}\left(\omega_{s}^{\prime}\right)\right. \\
& \left.+\int_{0}^{\infty} d \omega_{s}^{\prime} V_{F F}\left(\omega_{s}+\omega_{s}^{\prime}\right) \hat{a}_{s_{F}}^{\mathrm{in} \dagger}\left(\omega_{s}^{\prime}\right)\right] .
\end{aligned}
$$

The functions $U_{F F}(\omega)$ and $V_{F F}(\omega)$ are given by the inverse Fourier transform of the expressions in Eqs. (59) and can be found numerically.

\section{B. Corrugation in the second-subharmonic field only}

We consider only the forward-propagating fundamental field and quasi-phase-matching of the nonlinear interaction given by the condition $\delta_{n l, q}+\delta_{p}^{0} / 2=0$. The equations (55) can then be written in the following matrix form

$$
\frac{d}{d z}\left[\begin{array}{c}
\hat{A}_{s_{F}}(z, \tau) \\
\hat{A}_{s_{B}}(z, \tau) \\
\hat{A}_{s_{F}}^{\dagger}(z, \tau) \\
\hat{A}_{s_{B}}^{\dagger}(z, \tau)
\end{array}\right]=i M\left[\begin{array}{c}
\hat{A}_{s_{F}}(z, \tau) \\
\hat{A}_{s_{B}}(z, \tau) \\
\hat{A}_{s_{F}}^{\dagger}(z, \tau) \\
\hat{A}_{s_{B}}^{\dagger}(z, \tau)
\end{array}\right],
$$

$$
M(\tau)=\left[\begin{array}{cccc}
\delta_{s}^{0} / 2 & K_{s}^{0} & -4 i K_{F} & 0 \\
-K_{s}^{0 *} & -\delta_{s}^{0} / 2 & 0 & 0 \\
-4 i K_{F}^{*} & 0 & -\delta_{s}^{0} / 2 & -K_{s}^{0 *} \\
0 & 0 & K_{s}^{0} & \delta_{s}^{0} / 2
\end{array}\right]
$$

introducing the nonlinear coupling constant $K_{F}(\tau)=$ $K_{n l, q}^{0} A_{p_{F}}(0, \tau)$.

Eigenvalues $\lambda_{j}$ of matrix $M$ in Eq. (62) can be derived as follows:

$$
\begin{aligned}
& \lambda_{1,2}(\tau)=\sqrt{\left(\Delta_{s}^{0}\right)^{2}-8\left|K_{F}\right|^{2} \pm 4\left|K_{F}\right| \sqrt{4\left|K_{F}\right|^{2}+\left|K_{s}^{0}\right|^{2}}}, \\
& \lambda_{3,4}(\tau)=-\lambda_{1,2}(\tau) .
\end{aligned}
$$

These eigenvalues are real for $\left|K_{F}\right|<\Delta_{s}^{0} /(4 \sqrt{2})$ and describe an oscillatory solution. Two real and two pure imaginary eigenvalues are found for $\left|K_{F}\right|>\Delta_{s}^{0} /(4 \sqrt{2})$ reflecting the presence of amplified and attenuated components of the fields. Using the eigenvalues $\lambda_{j}$, the solution to Eqs. (62) can be written in a general form using operator constants $\hat{\alpha}_{j}$ and $\hat{\beta}_{j}$,

$$
\begin{aligned}
\hat{A}_{s_{F}}(z, \tau) & =\sum_{j=1}^{4} \hat{\alpha}_{j}(\tau) \exp \left[i \lambda_{j}(\tau) z\right], \\
\hat{A}_{s_{B}}(z, \tau) & =\sum_{j=1}^{4} \hat{\beta}_{j}(\tau) \exp \left[i \lambda_{j}(\tau) z\right] .
\end{aligned}
$$

Substitution of the general solution (64) into the second (or the fourth) equation in (62) provides the relations giving the coefficients $\hat{\beta}_{j}$ in terms of the coefficients $\hat{\alpha}_{j}$,

$$
\hat{\beta}_{j}=-\frac{K_{s}^{0 *}}{\lambda_{j}+\delta_{s}^{0} / 2} \hat{\alpha}_{j}, \quad j=1, \ldots, 4 .
$$

Assuming real eigenvalues $\lambda_{j}$ the first (or the third) equation in (62) is fulfilled provided that

$$
\begin{aligned}
\hat{\alpha}_{3,4} & =\gamma_{1,2} \hat{\alpha}_{1,2}^{\dagger}, \\
\gamma_{1,2} & =-\frac{4 i K_{F}\left(\lambda_{3,4}+\delta_{s}^{0} / 2\right)}{\lambda_{3,4}^{2}-\left(\delta_{s}^{0}\right)^{2} / 4+\left|K_{s}^{0}\right|^{2}} .
\end{aligned}
$$

The operator constants $\hat{\alpha}_{1}, \hat{\alpha}_{2}, \hat{\alpha}_{1}^{\dagger}$ and $\hat{\alpha}_{2}^{\dagger}$ are finally determined from the boundary conditions that give us the following formulas:

$$
\begin{gathered}
{\left[\begin{array}{c}
\hat{\alpha}_{1}(\tau) \\
\hat{\alpha}_{2}(\tau) \\
\hat{\alpha}_{1}^{\dagger}(\tau) \\
\hat{\alpha}_{2}^{\dagger}(\tau)
\end{array}\right]=M_{1}^{-1}(\tau)\left[\begin{array}{c}
\hat{A}_{s_{F}}(0, \tau) \\
\hat{A}_{s_{B}}(L, \tau) \\
\hat{A}_{s_{F}}^{\dagger}(0, \tau) \\
\hat{A}_{s_{B}}^{\dagger}(L, \tau)
\end{array}\right],} \\
M_{1}(\tau)=\left[\begin{array}{cccc}
1 & 1 & \gamma_{1} & \gamma_{2} \\
\vartheta_{1} & \vartheta_{2} & \vartheta_{3} \gamma_{1} & \vartheta_{4} \gamma_{2} \\
\gamma_{1}^{*} & \gamma_{2}^{*} & 1 & 1 \\
\vartheta_{3}^{*} \gamma_{1}^{*} & \vartheta_{4}^{*} \gamma_{2}^{*} & \vartheta_{1}^{*} & \vartheta_{2}^{*}
\end{array}\right] ; \\
\vartheta_{j}=-K_{s}^{0 *} /\left(\lambda_{j}+\delta_{s}^{0} / 2\right) \exp \left(i \lambda_{j} L\right) .
\end{gathered}
$$


Using Eqs. (64) and (65) the solution for the output operators can be written as

$$
\begin{aligned}
& \hat{A}_{s_{F}}(L, \tau)=\sum_{j=1}^{4} \hat{\alpha}_{j}(\tau) \exp \left[i \lambda_{j}(\tau) L\right], \\
& \hat{A}_{s_{B}}(0, \tau)=-\sum_{j=1}^{4} \frac{K_{s}^{0 *}}{\lambda_{j}(\tau)+\delta_{s}^{0} / 2} \hat{\alpha}_{j}(\tau) .
\end{aligned}
$$

The inverse Fourier transform of the formulas in Eq. (68) and return to the original operators $\hat{a}_{s_{F}}$ and $\hat{a}_{s_{B}}$ leaves us with the input-output relations written in the form:

$$
\begin{gathered}
\hat{a}_{s_{b}}^{\text {out }}\left(\omega_{s}\right)=\sum_{c=F, B}\left[\int_{0}^{\infty} d \omega_{s}^{\prime} U_{b c}\left(\omega_{s}-\omega_{s}^{\prime}\right) \hat{a}_{s_{c}}^{\text {in }}\left(\omega_{s}^{\prime}\right)\right. \\
\left.+\int_{0}^{\infty} d \omega_{s}^{\prime} V_{b c}\left(\omega_{s}+\omega_{s}^{\prime}\right) \hat{a}_{s_{c}}^{\text {in } \dagger}\left(\omega_{s}^{\prime}\right)\right], \quad b=F, B .
\end{gathered}
$$

The matrices $U_{b c}$ and $V_{b c}$ in Eq. (69) can be determined numerically in general.

\section{GENERAL NUMERICAL SOLUTION AND THE BLOCH-MESSIAH REDUCTION}

To investigate the model numerically we have to replace Eqs. (8) for the SSF field by their discrete variants. That is why we introduce discrete monochromatic-mode operator amplitudes $\hat{A}_{s_{b}, i}$ that scan the spectral profiles with period $\Delta \omega$,

$$
\begin{aligned}
\hat{A}_{s_{b}, i}(z)=\sqrt{\Delta \omega} & \hat{A}_{a_{b}}\left(z, \omega_{s}^{0}+i \Delta \omega\right), \\
& b=F, B, \quad i=0, \pm 1 \pm 2, \ldots
\end{aligned}
$$

The discrete mode operator amplitudes $\hat{A}_{a_{b}, i}$ obey the usual boson commutation relations instead of those written in Eq. (6). These operator amplitudes can be ordered into vectors $\hat{\mathbf{A}}_{s_{F}}$ and $\hat{\mathbf{A}}_{s_{B}}$. Using these vectors the discrete form of Eqs. (8) can be written as follows:

$$
\begin{aligned}
& d \frac{d}{d z}\left[\begin{array}{c}
\hat{\mathbf{A}}_{s_{F}}(z) \\
\hat{\mathbf{A}}_{s_{F}}^{\dagger}(z) \\
\hat{\mathbf{A}}_{s_{B}}(z) \\
\hat{\mathbf{A}}_{s_{B}}^{\dagger}(z)
\end{array}\right]=i \mathcal{M}(z)\left[\begin{array}{c}
\hat{\mathbf{A}}_{s_{F}}(z) \\
\hat{\mathbf{A}}_{s_{F}}^{\dagger}(z) \\
\hat{\mathbf{A}}_{s_{B}}(z) \\
\hat{\mathbf{A}}_{s_{B}}^{\dagger}(z)
\end{array}\right], \\
& \mathcal{M}(z)=\left[\begin{array}{cccc}
\mathcal{D}_{s} & -4 i \mathcal{K}_{s F}(z) & \mathcal{K}_{s} & 0 \\
-4 i \mathcal{K}_{s F}^{*}(z) & -\mathcal{D}_{s} & 0 & -\mathcal{K}_{s}^{*} \\
-\mathcal{K}_{s}^{*} & 0 & -\mathcal{D}_{s} & 4 i \mathcal{K}_{s B}(z) \\
0 & \mathcal{K}_{s} & 4 i \mathcal{K}_{s B}^{*}(z) & \mathcal{D}_{s}
\end{array}\right] .
\end{aligned}
$$

Matrix elements of the matrices $\mathcal{D}_{s}, \mathcal{K}_{s}, \mathcal{K}_{s F}(z)$, and $\mathcal{K}_{s B}(z)$ are defined as

$$
\mathcal{D}_{s, j k}=\delta_{j k} \frac{\delta_{s}\left(\omega_{s}^{0}+k \Delta \omega\right)}{2},
$$

$$
\begin{gathered}
\mathcal{K}_{s, j k}=\delta_{j k} K_{s}\left(\omega_{s}^{0}+k \Delta \omega\right), \\
\mathcal{K}_{s F, j k}(z)=\Delta \omega K_{n l, q}\left(\omega_{s}^{0}+j \Delta \omega, \omega_{s}^{0}+k \Delta \omega\right) \\
\times \exp \left(i \delta_{n l, q} z\right) A_{p_{F}}\left[z, \omega_{p}^{0}+(j+k) \Delta \omega\right], \\
\mathcal{K}_{s B, j k}(z)=\Delta \omega K_{n l, q}\left(\omega_{s}^{0}+j \Delta \omega, \omega_{s}^{0}+k \Delta \omega\right) \\
\times \exp \left(-i \delta_{n l, q} z\right) A_{p_{B}}\left[z, \omega_{p}^{0}+(j+k) \Delta \omega\right],
\end{gathered}
$$

where $\delta_{j k}$ means the Kronecker symbol.

As the matrix $\mathcal{M}$ in Eq. (71) depends on $z$, only numerical solution is possible in general. We also need to keep quantum features in the solution and so we have to solve the system of linear equations (71) for initial vectors that form a basis. In this way, we reveal the whole evolution matrix $\mathcal{U}$ that maps the operator fields at $z=0$ to those at $z=L$ :

$$
\begin{gathered}
{\left[\begin{array}{c}
\hat{\mathbf{A}}_{s_{F}}(L) \\
\hat{\mathbf{A}}_{s_{F}}^{\dagger}(L) \\
\hat{\mathbf{A}}_{s_{B}}(L) \\
\hat{\mathbf{A}}_{s_{B}}^{\dagger}(L)
\end{array}\right]=\mathcal{U}\left[\begin{array}{c}
\hat{\mathbf{A}}_{s_{F}}(0) \\
\hat{\mathbf{A}}_{s_{F}}^{\dagger}(0) \\
\hat{\mathbf{A}}_{s_{B}}(0) \\
\hat{\mathbf{A}}_{s_{B}}^{\dagger}(0)
\end{array}\right],} \\
\mathcal{U}=\left[\begin{array}{ll}
\mathcal{U}_{F F} & \mathcal{U}_{F B} \\
\mathcal{U}_{B F} & \mathcal{U}_{B B}
\end{array}\right] .
\end{gathered}
$$

We note that the operator amplitudes contained in vectors $\hat{\mathbf{A}}_{s_{F}}(L)$ and $\hat{\mathbf{A}}_{s_{B}}(L)$ obey certain kind of commutation relations useful in the numerical solution (for details, see [37]).

Partial inversion of the linear relations in Eq. (73) reveals the input-output relations among the operator amplitudes,

$$
\begin{gathered}
{\left[\begin{array}{c}
\hat{\mathbf{A}}_{s_{F}}(L) \\
\hat{\mathbf{A}}_{s_{F}}^{\dagger}(L) \\
\hat{\mathbf{A}}_{s_{B}}(0) \\
\hat{\mathbf{A}}_{s_{B}}^{\dagger}(0)
\end{array}\right]=\mathcal{U}^{\text {pinv }}\left[\begin{array}{c}
\hat{\mathbf{A}}_{s_{F}}(0) \\
\hat{\mathbf{A}}_{s_{F}}^{\dagger}(0) \\
\hat{\mathbf{A}}_{s_{B}}(L) \\
\hat{\mathbf{A}}_{s_{B}}^{\dagger}(L)
\end{array}\right],} \\
\mathcal{U}^{\text {pinv }}=\left[\begin{array}{cc}
\mathcal{U}_{F F}-\mathcal{U}_{F B} \mathcal{U}_{B B}^{-1} \mathcal{U}_{B F} & \mathcal{U}_{F B} \mathcal{U}_{B B}^{-1} \\
-\mathcal{U}_{B B}^{-1} \mathcal{U}_{B F} & \mathcal{U}_{B B}^{-1}
\end{array}\right] .
\end{gathered}
$$

The output operator amplitudes in the vectors $\hat{\mathbf{A}}_{s_{F}}(L)$ and $\hat{\mathbf{A}}_{s_{B}}(0)$ obey the boson commutation relations provided that the input operator amplitudes given in the vectors $\hat{\mathbf{A}}_{s_{F}}(0)$ and $\hat{\mathbf{A}}_{s_{B}}(L)$ fulfil boson commutation relations. That is why it is convenient to rewrite the relations in Eq. (74) into the form of Bogoljubov transformation. Using the operators $\hat{a}_{s_{b}, i}^{\text {in }}$ and $\hat{a}_{s_{b}, i}^{\text {out }}$ defined in analogy with their continuous counterparts we have:

$$
\left[\begin{array}{l}
\hat{\mathbf{a}}_{s F}^{\text {out }} \\
\hat{\mathbf{a}}_{s_{B}}^{\text {out }}
\end{array}\right]=\mathbf{U}\left[\begin{array}{c}
\hat{\mathbf{a}}_{s_{F}}^{\text {in }} \\
\hat{\mathbf{a}}_{s_{B}}^{\mathrm{in}}
\end{array}\right]+\mathbf{V}\left[\begin{array}{c}
\hat{\mathbf{a}}_{s_{F}}^{\mathrm{in}} \\
\hat{\mathbf{a}}_{s_{B}}^{\mathrm{in}}
\end{array}\right]
$$

The vectors $\hat{\mathbf{a}}_{s_{b}}^{\text {in }}$ and $\hat{\mathbf{a}}_{s_{b}}^{\text {out }}(b=F, B)$ are composed of the operator amplitudes $\hat{a}_{s_{b}, i}^{\text {in }}$ and $\hat{a}_{s_{b}, i}^{\text {out }}$, respectively.

As the matrices $\mathbf{U}$ and $\mathbf{V}$ describe the Bogoljubov transformation, their Bloch-Messiah reduction [48, 49, 62] can be found,

$$
\begin{aligned}
\mathbf{U} & =\mathbf{X} \boldsymbol{\Lambda}_{U} \mathbf{Y}^{\dagger}, \\
\mathbf{V} & =\mathbf{X} \boldsymbol{\Lambda}_{V} \mathbf{Y}^{T},
\end{aligned}
$$


where $\dagger$ means the hermitian conjugation and $T$ stands for the matrix transposition. The matrices $\boldsymbol{\Lambda}_{U}$ and $\boldsymbol{\Lambda}_{V}$ are diagonal and contain real nonnegative eigenvalues of the decomposition. The matrix $\mathbf{Y}(\mathbf{X})$ in Eq. (76) contains the right (left) eigenvectors $\mathbf{Y}_{i}\left(\mathbf{X}_{i}\right)$.

The eigenvectors defined by the Bloch-Messiah reduction give typical modes of the nonlinear interaction and represent a discrete form of eigenmode spectral functions $\phi_{n}$ found in Eq. (35) in the analytical perturbation approach.

Coefficients $B_{s, n}$ and $C_{s, n}$ of the generalized superposition of signal and noise [9] written for an $n$-th eigenmode of the Bloch-Messiah reduction (76) and defined in Eqs. (49) can be expressed using the eigenvalues of the decomposition,

$$
\begin{aligned}
B_{s, n}= & \boldsymbol{\Lambda}_{U, n n}^{2}\left(B_{s, n \mathcal{A}}^{\mathrm{in}}-1\right)+\boldsymbol{\Lambda}_{V, n n}^{2} B_{s, n \mathcal{A}}^{\mathrm{in}} \\
& +\left(\boldsymbol{\Lambda}_{U, n n} \boldsymbol{\Lambda}_{V, n n} C_{s, n \mathcal{A}}^{\mathrm{in}}+\text { c.c. }\right), \\
C_{s, n}= & \boldsymbol{\Lambda}_{U, n n}^{2} C_{s, n \mathcal{A}}^{\mathrm{in}}+\boldsymbol{\Lambda}_{V, n n}^{2} C_{s, n \mathcal{A}}^{\mathrm{in} *} \\
& +\boldsymbol{\Lambda}_{U, n n} \boldsymbol{\Lambda}_{V, n n}\left(2 B_{s, n \mathcal{A}}^{\mathrm{in}}-1\right) .
\end{aligned}
$$

Symbol c.c. stands for the complex conjugated term. The coefficients $B_{s, n \mathcal{A}}^{\text {in }}$ and $C_{s, n \mathcal{A}}^{\text {in }}$ related to anti-normal operator ordering characterize the incident field of eigenmode $n$ and can be written as

$$
\begin{aligned}
B_{s, n \mathcal{A}}^{\text {in }} & =\cosh ^{2}\left(r_{n}\right)+n_{n, \text { noise }} \\
C_{s, n \mathcal{A}}^{\text {in }} & =\frac{\exp \left(i \theta_{n}\right) \sinh \left(2 r_{n}\right)}{2}
\end{aligned}
$$

where $r_{n}$ is the squeeze parameter, $\theta_{n}$ the squeeze phase, and $n_{n, \text { noise }}$ the mean number of noisy photons in eigenmode $n$. The number $N_{s, n}$ of photons in eigenmode $n$ $\left[N=\left\langle\hat{a}^{\dagger} \hat{a}\right\rangle\right]$ attains a simple form:

$$
N_{s, n}=\left|\xi_{s, n}\right|^{2}+B_{s, n}
$$

where $\xi_{s, n}$ gives the initial coherent amplitude in eigenmode $n$. The principal squeeze variance $\lambda_{s, n}$ of eigenmode $n$ is obtained by the formula analogous to that in Eq. (48) above.

The importance of the Bloch-Messiah reduction in the investigation of squeezing is emphasized by the fact that an eigenmode with the lowest value of the principal squeeze variance $\lambda_{s_{F}, n}$ represents the solution of the optimization problem for a suitable spectral mode profile that gives the best possible amount of squeezing (see Appendix A).

In the experiment, either forward- or backwardpropagating fields are interesting. Their properties can be obtained if we decompose the eigenvectors $\mathbf{X}_{n}$ of the Bloch-Messiah reduction into their mutually orthogonal forward- $\left(\mathbf{X}_{F, n}\right)$ and backward- $\left(\mathbf{X}_{B, n}\right)$ propagating parts. The corresponding principal squeeze variances $\lambda_{s_{b}, n}$ and mean photon numbers $N_{s_{b}, n}$ are given by the weighted sums of the quantities related to the eigenvectors of the original Bloch-Messiah reduction,

$$
\lambda_{s_{b}, n}=\sum_{n^{\prime}} c_{b, n n^{\prime}} \lambda_{s, n^{\prime}}
$$

$$
N_{s_{b}, n}=\sum_{n^{\prime}} c_{b, n n^{\prime}} N_{s, n^{\prime}}, \quad b=F, B
$$

Using the scalar product $\cdot$ the coefficients $c_{b, n n^{\prime}}$ are given as

$$
c_{b, n n^{\prime}}=\frac{\left|\mathbf{X}_{b, n}^{\dagger} \cdot \mathbf{X}_{n^{\prime}}\right|^{2}}{\mathbf{X}_{b, n}^{\dagger} \cdot \mathbf{X}_{b, n}}, \quad b=F, B
$$

The number of effectively populated modes belongs to the most important characteristics of the SSF field. It can be obtained from the analysis of the amplitude correlation functions $\left\langle\hat{a}_{s, n}^{\text {out }} \hat{a}_{s, n^{\prime}}^{\text {out }}\right\rangle_{\text {vac }}$ giving the correlations between the amplitudes $\hat{a}_{s, n}^{\text {out }}$ associated with the eigenmodes of the Bloch-Messiah reduction. For simplicity, the correlation functions are defined for the incident vacuum state |\rangle$_{\text {vac }}$ in the SSF field. Suitability of these correlation functions for the determination of the number of effective modes originates in the fact that it describes paired photons in the SSF field. These paired photons are, according to the formula for momentum operator $\hat{G}$ in Eq. (3), the elementary entities characterizing the process of second-subharmonic generation. Using the Bloch-Messiah decomposition in Eq. (76) the amplitude correlation functions can be written as:

$$
\left\langle\hat{a}_{s, n}^{\text {out }} \hat{a}_{s, n^{\prime}}^{\text {out }}\right\rangle_{\text {vac }}=\delta_{n n^{\prime}} \mathbf{X}_{n} \boldsymbol{\Lambda}_{V, n n} \boldsymbol{\Lambda}_{U, n n} \mathbf{X}_{n}^{T}
$$

According to Eq. (82) the real number $\boldsymbol{\Lambda}_{V, n n} \boldsymbol{\Lambda}_{U, n n}$ determines the weight of the contribution of an $n$-th eigenmode. After proper renormalization of these weights guaranteeing $\sum_{n}\left(\boldsymbol{\Lambda}_{V, n n} \boldsymbol{\Lambda}_{U, n n}\right)^{2}=1$, the number $K$ of effectively populated modes is given by the cooperativity parameter,

$$
K=\frac{\left[\sum_{n}\left(\boldsymbol{\Lambda}_{V, n n} \boldsymbol{\Lambda}_{U, n n}\right)^{2}\right]^{2}}{\sum_{n}\left(\boldsymbol{\Lambda}_{V, n n} \boldsymbol{\Lambda}_{U, n n}\right)^{4}} .
$$

Monochromatic frequency modes play dominant role in the experimentally determined quantities. They can be easily evaluated using the matrices $\mathbf{U}$ and $\mathbf{V}$ occurring in the solution in Eq. (75). For example and assuming the incident vacuum state in the SSF field, the amplitude frequency correlation function $N_{s_{b} s_{b^{\prime}}, \omega}\left(\omega_{s, j}, \omega_{s, j^{\prime}}\right)$ is determined along the expression

$$
\begin{gathered}
N_{s_{b} s_{b^{\prime}}, \omega}\left(\omega_{s, j}, \omega_{s, j^{\prime}}\right)=\left\langle\hat{a}_{s_{b}}^{\text {out } \dagger}\left(\omega_{s, j}\right) \hat{a}_{s_{b^{\prime}}}^{\text {out }}\left(\omega_{s, j^{\prime}}\right)\right\rangle_{\mathrm{vac}} \\
=\frac{1}{\Delta \omega} \sum_{d=F, B} \sum_{k} V_{b d, j k}^{*} V_{b^{\prime} d, j^{\prime} k}, \quad b, b^{\prime}=F, B ;
\end{gathered}
$$

$\omega_{s, j}=\omega_{s}^{0}+j \Delta \omega$. The matrices $V_{b d}$ introduced in Eq. 84 [and similarly the matrices $U_{b d}$ used later] are obtained from the matrix $\mathbf{V}[\mathbf{U}]$ in Eq. (75) by grouping its matrix elements with respect to the propagation direction. The spectral photon-number density in mode $b$ is given by the quantity $N_{s_{b} s_{b}, \omega}^{d}\left(\omega_{s, j}, \omega_{s, j}\right)$.

In the time domain, amplitude correlations at two instants $\tau$ and $\tau^{\prime}$ are characterized by the temporal ampli- 
tude correlation function $N_{s_{b} s_{b^{\prime}}, \tau}\left(\tau, \tau^{\prime}\right)$ expressed as

$$
\begin{aligned}
N_{s_{b} s_{b^{\prime}}, \tau}\left(\tau, \tau^{\prime}\right)= & \int d \omega_{s} \int d \omega_{s}^{\prime} \exp \left(i \omega_{s} \tau\right) \exp \left(-i \omega_{s}^{\prime} \tau^{\prime}\right) \\
& \times N_{s_{b} s_{b^{\prime}}, \omega}\left(\omega_{s}, \omega_{s}^{\prime}\right) \\
= & (\Delta \omega)^{2} \sum_{j, j^{\prime}} \exp \left(i \omega_{s, j} \tau\right) \exp \left(-i \omega_{s, j^{\prime}} \tau^{\prime}\right) \\
& \times N_{s_{b} s_{b^{\prime}}, \omega}\left(\omega_{s, j}, \omega_{s, j^{\prime}}\right)
\end{aligned}
$$

For $\tau=\tau^{\prime}$, the quantity in Eq. 855) gives the flux expressed in photon numbers.

\section{DISCUSSION OF THE PULSED SQUEEZED-LIGHT GENERATION}

We assume that the incident forward-propagating fundamental field is given by a Gaussian ultrashort pulse with the central wavelength $\lambda_{p}^{0}=532 \times 10^{-9} \mathrm{~m}$ and pulse duration $\tau_{p}=1 \times 10^{-13} \mathrm{~s}$ [see Eq. (25)] originating in the second-harmonic frequency generation from a pulsed Nd:YAG laser. Knowing its incident power $P_{p_{F}}$ and repetition rate $f\left(f=1 \times 10^{8} \mathrm{~s}^{-1}\right)$, the incident amplitude $\xi_{p}$ defined in Eq. (25) is obtained by the formula

$$
\xi_{p}=\sqrt{\frac{P_{p_{F}} L \beta_{p}^{0}}{\hbar\left(\omega_{p}^{0}\right)^{2} f}} .
$$

Waveguide's depth $t$ equals $5 \times 10^{-7} \mathrm{~m}$. Its width $\Delta y$ is $1 \times 10^{-6} \mathrm{~m}$. Depth $t_{l}$ of the periodic corrugation is $5 \times 10^{-8} \mathrm{~m}$ which guarantees single-mode operation at the studied frequencies (for more details, see [28]). Period $\Lambda_{l}$ of this corrugation is determined by Eq. (10) such that the scattered field $a$ fulfills the resonant condition $\Delta_{a}^{0}=$ $m \pi / L$ for the first transmission peak $(m=1)$, i.e. $\delta_{a}^{0}=$ $\pm \sqrt{\pi^{2} / L^{2}+\left|K_{a}^{0}\right|^{2}}$. The natural quasi-phase mismatch $\delta_{n l, q}^{\mathrm{nat}, 0}$ is then given in Eq. (44) for the fundamental field and Eq. (47) for the SSF field and determines the period $\Lambda_{n l}$ of nonlinear periodic poling (for details, see the end of Subsec. IIIB). We consider two different waveguide's lengths, $L=1 \times 10^{-3} \mathrm{~m}$ and $L=1 \times 10^{-2} \mathrm{~m}$, in the discussion. Spectral and modal properties of the SSF field are conveniently discussed in the shorter waveguide, that provides wider SSF spectra. On the other hand, the nonlinear interaction is sufficiently developed in the longer waveguide which results in useful values of the principal squeeze variance $\lambda$.

The section is divided into two parts. In the first part spectral modes and their structure are discussed using the simplified model with non-dispersion propagation developed in Sec. IV. General discussion of the behavior of the SSF field as it arises from numerical solution of the model of Sec. V is contained in the second part. a)

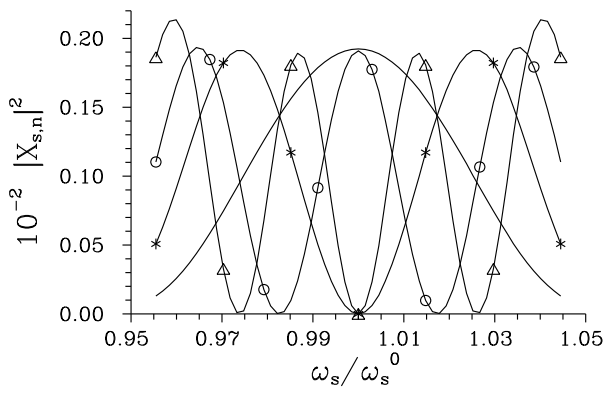

b)

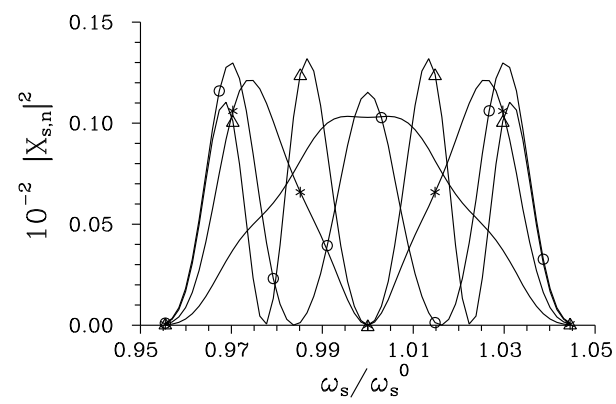

FIG. 5: Intensity spectral profiles $\left|\mathbf{X}_{s, n}\right|^{2}$ for the scattered (a) fundamental and (b) SSF field for the first (plane solid curve), second (solid curve with $*$ ), third (solid curve with $\circ$ ), and fourth (solid curve with $\triangle$ ) eigenmode obtained in the model with non-dispersion propagation. The profiles are normalized such that $\int d \omega\left|\mathbf{X}_{s, n}(\omega)\right|^{2} / \omega_{s}^{0}=1$. In (a) $\Lambda_{l}=1.151 \times 10^{-7} \mathrm{~m}$, $\Lambda_{n l}=3.5510 \times 10^{-6} \mathrm{~m}$ and in (b) $\Lambda_{l}=2.459 \times 10^{-7} \mathrm{~m}$, $\Lambda_{n l}=3.5547 \times 10^{-6} \mathrm{~m} ; P_{p_{F}}=1 \times 10^{-6} \mathrm{~W}, L=1 \times 10^{-3} \mathrm{~m}$.

\section{A. Spectral modes and their properties}

The simplified model with non-dispersion propagation is useful namely in revealing spectral properties of eigenmodes of the nonlinear interaction. When the fundamental-field scattering is considered, the SSF-field eigenmodes maintain qualitatively the features obtained in the perturbation Gaussian approach in Sec. III resulting in the formula (35) (see also [47, 50]). Thus, an $n$-th eigenmode $(n=1,2, \ldots)$ has $n-1$ zeroes in its intensity profile [see Fig. [5(a)]. Also, the larger the number $n$ of eigenmode, the wider the mode is.

The eigenmode structure is more complex for the scattered SSF field that has the forward- and backwardpropagating components. Intensity profiles of these components in one eigenmode are the same. Moreover, there exist pairs of eigenmodes with the same intensity profile. However, they are mutually orthogonal due to their different spectral phase profiles. Also here intensity profiles of the components have $n-1$ zeros for an $n$-th pair of eigenmodes [see Fig. [5(b)].

The absence of inter-mode dispersion leads to the fact that spectral widths of eigenmodes are given by the bandwidth of a frequency filter used in the experiment. The fact that the propagating monochromatic waves of the SSF field are in phase effectively increases the nonlinear interaction. As a consequence, smaller values of princi- 


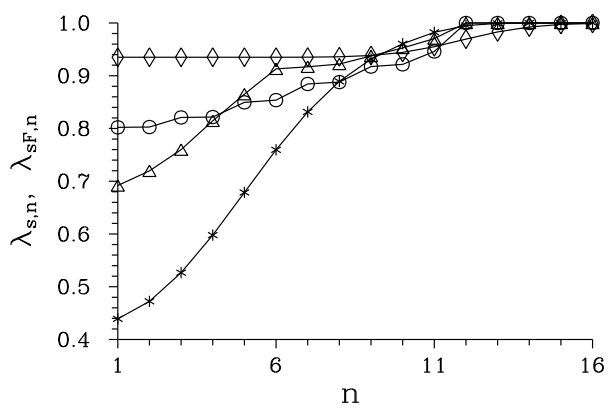

FIG. 6: Principal squeeze variances $\lambda_{s, n}$ of the maximumsqueezed 15 eigenmodes for the scattered fundamental (solid curve with $*$ ) and SSF (solid curve with $\triangle$ ) field determined assuming non-dispersion propagation. Also the principal squeeze variances $\lambda_{s_{F}, n}$ of the forward-propagating SSF field are shown (solid curve with o). For comparison, principal squeeze variances $\lambda_{s, n}$ of the real waveguide without the scattered fields (solid curve with $\diamond$ ) are drawn; $P_{p_{F}}=1 \times 10^{-6} \mathrm{~W}$, $L=1 \times 10^{-3} \mathrm{~m}$.

pal squeeze variances $\lambda_{s, n}$ and greater SSF-field photon numbers $N_{s, n}$ compared to the real ones are predicted in the model. The principal squeeze variances $\lambda_{s, n}$ of the first 15 eigenmodes are drawn in Fig. 6 for the scattered fundamental and SSF fields. The principal squeeze variances $\lambda_{s, n}$ for the fundamental-field scattering are smaller than those for the SSF-field scattering because the scattering in the SSF field is weaker [compare the curves in Figs. 2(a) and 3(a)]. In case of the scattered SSF field, even the principal squeeze variances $\lambda_{s_{F}, n}$ characterizing the forward-propagating field and given by the formula (80) are shown. The values of variances $\lambda_{s_{F}, n}$ can clearly be grouped into pairs which originates in pairing of eigenmodes discussed above. However, we note that the modes of the forward-propagating SSF field arising from the decomposition of eigenmodes into their forwardand backward-propagating components are not mutually orthogonal. In fact, the number of such modes is twice to that given by the dimension of the appropriate space. The comparison of values of principal squeeze variances $\lambda_{s, n}$ obtained for the waveguide with and without scattering reveals substantial improvement caused by the scattering (see Fig. 6).

\section{B. Pulsed squeezed-light generation}

We analyze the general solution using the model of Sec. V assuming the fundamental-field scattering and compare the obtained results with those appropriate for the waveguide without scattering. Considering the shorter waveguide, the intensity spectral profiles of the first four eigenmodes are plotted in Fig. 7 for both cases. Compared to the profiles of the model without dispersion shown in Fig. [5(a) the obtained intensity spectral profiles are naturally bounded by spectral properties of the waveguide (material and waveguiding dispersion, see a)

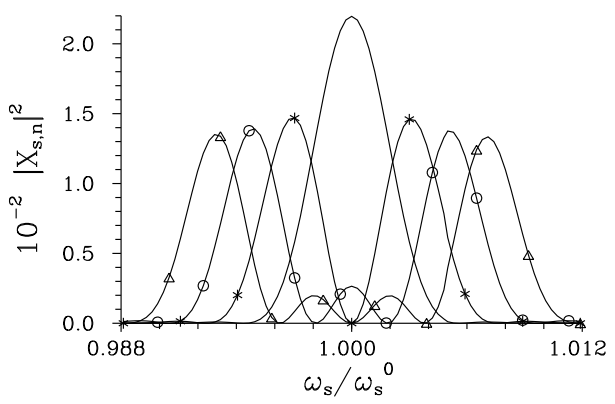

b)

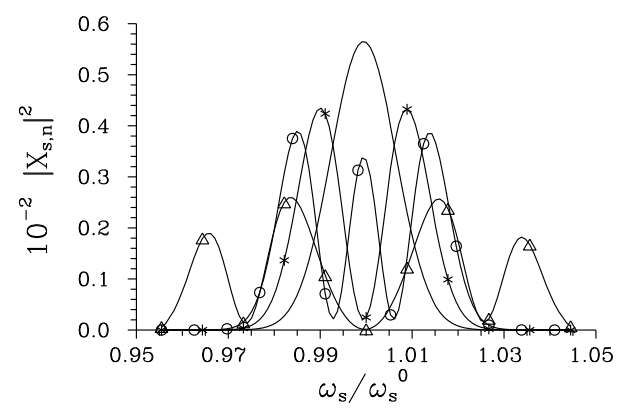

FIG. 7: Intensity spectral profiles $\left|\mathbf{X}_{s, n}\right|^{2}$ for the first (solid curve without symbols), second (solid curve with $*$ ), third (solid curve with o), and fourth (solid curve with $\triangle$ ) SSFfield eigenmode for (a) no scattered field and (b) scattered fundamental field. In (a) $\Lambda_{n l}=3.5516 \times 10^{-6} \mathrm{~m}$ and in (b) $\Lambda_{l}=1.151 \times 10^{-7} \mathrm{~m}, \Lambda_{n l}=3.5510 \times 10^{-6} \mathrm{~m} ; P_{p_{F}}=$ $1 \times 10^{-6} \mathrm{~W}, L=1 \times 10^{-3} \mathrm{~m}$.

Fig. (7). Whereas the intensity spectral profiles maintain the appropriate number of zeros in the case without scattering, scattering of the fundamental field leads to the replacement of zeros by nonzero minima in these profiles. Scattering in the fundamental field considerably broadens eigenmode spectral profiles [compare Figs. Z(a) and (b)] on one side, on the other side it makes the overall spectra narrower [see Fig. 9(a) below]. This is caused by a complex phase structure of the generated SSF field that requires a greater number of eigenmodes in its decomposition. As these eigenmodes have to be mutually orthogonal, their spectra have to be wider. The mode structure does not significantly change when the incident power of the fundamental field increases. This is caused by the approximation assuming a non-depleted fundamental field.

On the other hand, increasing values of the incident fundamental-field power $P_{p_{F}}$ decrease the principal squeeze variances $\lambda_{s, n}$ in all eigenmodes. This is documented in Fig. 8 showing the variances $\lambda_{s, n}$ for three different values of the power $P_{p_{F}}$. Comparison of the curves in Figs. 8(a) and (b) allows to judge the effectiveness of scattering in the fundamental field from the point of view of squeezed-light generation. Scattering of the fundamental field not only increases squeezing in the eigenmodes, it also considerably increases the number $K$ of effectively populated eigenmodes. Whereas $K \approx 11$ for the case without scattering, $K \approx 49$ is found for the 
a)

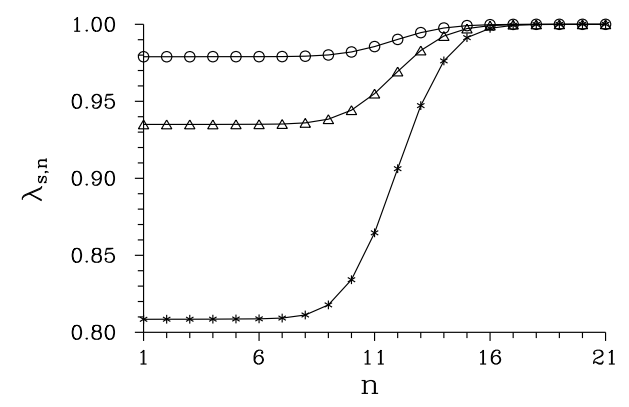

b)

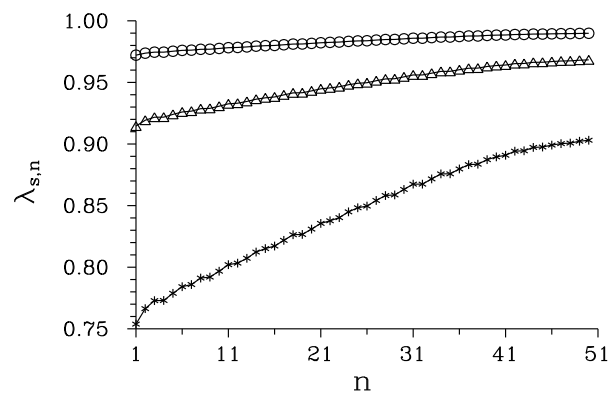

FIG. 8: Principal squeeze variances $\lambda_{s, n}$ depending on the mode number $n$ for $P_{p_{F}}=1 \times 10^{-7} \mathrm{~W}$ (solid curve with $\circ$ ), $P_{p_{F}}=1 \times 10^{-6} \mathrm{~W}$ ( solid curve with $\triangle$ ), and $P_{p_{F}}=1 \times 10^{-5} \mathrm{~W}$ (solid curve with $*$ ) for (a) no scattered field and (b) scattered fundamental field. Values of the parameters are the same as in the caption to Fig. 7

scattered fundamental field. Larger values of the number $K$ of effectively populated modes are important for pulsed homodyne detection [7] as they lower the requirements to the amplitude profile of the used local-oscillator field.

As the nonlinear interaction populates a larger number of eigenmodes, the intensity spectrum $N_{s s, \omega}^{d}$ of the overall SSF field is relatively wide [see Fig. 9(a)]. This is caused by nearly linear spectral dependencies of the linear phase mismatches [see the curves in Figs. 2(b) and 3(b)]. On the other hand, amplitude spectral correlations given mainly by the fundamental-field spectral width are narrow for both considered cases [see Fig. 9(b) for a cut across the correlation function $\left.N_{s s, \omega}\left(\omega_{s}, \omega_{s}^{\prime}\right)\right]$. Also spectral oscillations originating in dispersion evolution along the $z$ axis can be found in these correlations.

The SSF field is generated in the form of an ultrashort pulse (see Fig. 10). Whereas its temporal profile is close to a rectangular shape for the case without scattering, its profile is broken into two parts when scattering of the fundamental field is considered. This scattering makes the SSF-field intensity spectra narrower and, as consequence, it also extends the SSF-field duration (from approx. $1.3 \mathrm{ps}$ to $2 \mathrm{ps}$ ). This extension of field duration is also caused by complex spectral phase relations imposed by scattering of the fundamental field. Splitting of the SSF-field pulse reflects spectral anti-symmetry around the central frequencies $\omega_{s}^{0}$ and $\omega_{p}^{0}$. Period $\Lambda_{l}$ of linear corrugation and period $\Lambda_{n l, 1}$ of nonlinear modulation are optimum only for the central frequencies. Whereas their a)

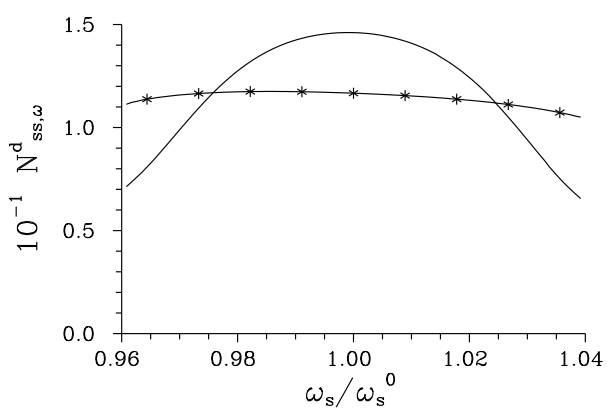

b)

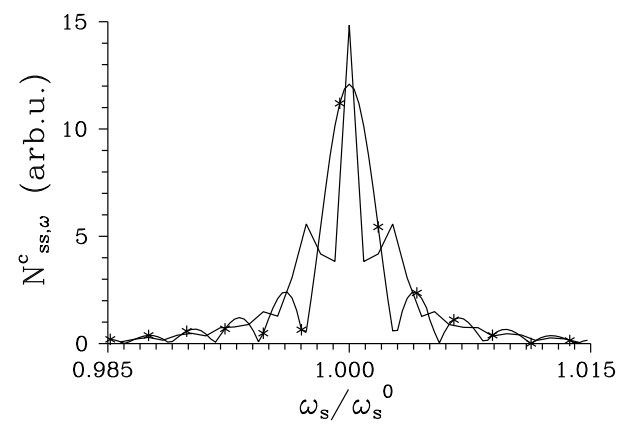

FIG. 9: (a) Intensity spectrum $N_{s s, \omega}^{d}(\omega) \equiv N_{s s, \omega}(\omega, \omega)$ and (b) cut $N_{s s, \omega}^{c}(\omega) \equiv\left|N_{s s, \omega}\left(\omega_{s}^{0}, \omega\right)\right|$ across the amplitude correlation function of the overall SSF field for the waveguide with the fundamental-field scattering (plane solid curve) and without scattering (solid curve with $*$ ). The spectra are normalized such that $\int d \omega N_{s s, \omega}^{d}(\omega) / \omega_{s}^{0}=1$. Values of the parameters are the same as in the caption to Fig. 7

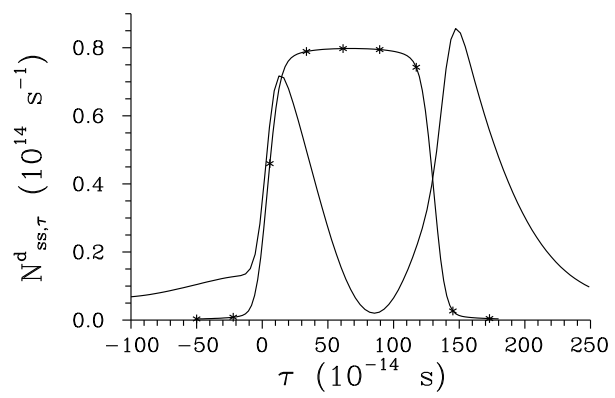

FIG. 10: Flux $N_{s s, \tau}^{d}(\tau) \equiv N_{s s, \tau}(\tau, \tau)$ of photon numbers in the SSF field for the waveguide with the fundamental-field scattering (plane solid curve) and without scattering (solid curve with $*$ ). It holds that $\int d \tau N_{s s, \tau}^{d}(\tau)=1$ and values of the parameters are the same as in the caption to Fig. 7.

values lead to insufficient compensation on one side of the spectrum, they overcompensate the nonlinear interaction on the other side of the spectrum. We note that the nearly rectangular shape in the case without scattering is given by the chosen values of fundamental-field pulse duration and waveguide length and their relation to the group velocities at the fundamental and SSF central frequencies.

In order to reach useful (i.e. sufficiently small) values of the principal squeeze variances $\lambda_{s, n}$, either greater fundamental-field powers or longer waveguides have to 
a)

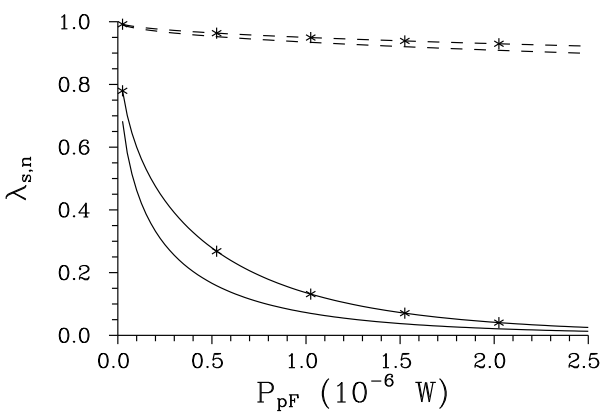

b)

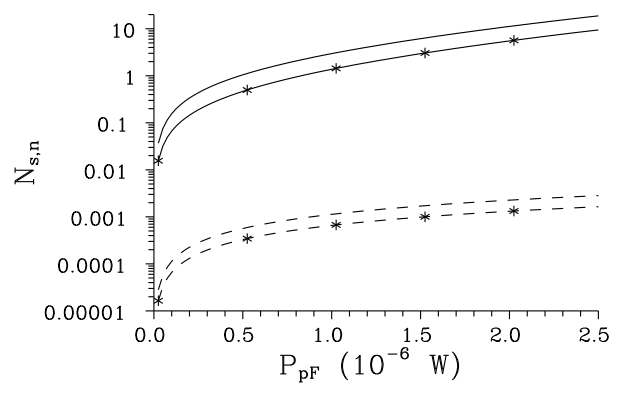

FIG. 11: (a) Principal squeeze variances $\lambda_{s, n}$ and (b) numbers $N_{s, n}$ of generated photons as they depend on incident fundamental-field power $P_{p_{F}}$. The quantities are shown for the waveguide with fundamental-field scattering $\left(\Lambda_{l}=\right.$ $1.151 \times 10^{-7} \mathrm{~m}, \Lambda_{n l}=3.5510 \times 10^{-6} \mathrm{~m}$, solid curves $)$ as well as without scattering $\left(\Lambda_{n l}=3.5516 \times 10^{-6} \mathrm{~m}\right.$, dashed curves). Plane curves are for $n=1$ whereas the curves with * are for $n=23$ (with scattering) and $n=14$ (without scattering) equal to the number $K$ of effectively populated modes; $L=1 \times 10^{-2} \mathrm{~m}$. In (b), the logarithmic $y$ axis is used.

be considered. Achievable values of the variances $\lambda_{s, n}$ as well as numbers $N_{s, n}$ of generated photons depend on the fundamental-field power and are plotted in Fig. 11 for the waveguide $10 \mathrm{~mm}$ long. As shown in Fig. 11 . small values of the variances $\lambda_{s, n}$ as well as greater values of photon numbers $N_{s, n}$ are practically found in all of the first $K$ eigenmodes. The improvement of squeezing caused by scattering in the fundamental field is dramatic [see Fig. 11(a)]. Scattering also increases the numbers $N_{s, n}$ of generated SSF-field photons by more than two orders in magnitude for the analyzed waveguide [see Fig. 11(b)]. As scattering also increases the number $K$ of effectively populated modes (from 14 to 23 ) the overall number $N_{s}$ of generated photons is nearly three orders in magnitude greater when the fundamental field is scattered (see Fig. 12).

Thus, a linear periodic corrugation with suitable parameters present in a nonlinear waveguide leads to important increase of effective nonlinearity that results in great improvement of amplitude squeezing of the generated light.

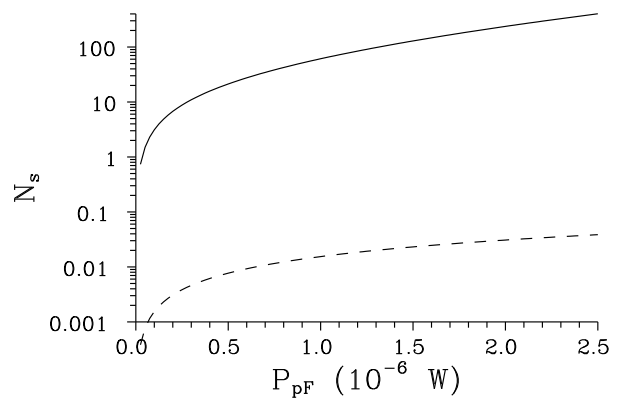

FIG. 12: Numbers $N_{s}$ of generated photons depending on incident fundamental-field power $P_{p_{F}}$ for the waveguide with fundamental-field scattering (solid curve) and without scattering (dashed curve); $L=1 \times 10^{-2} \mathrm{~m}$. The logarithmic $y$ axis is used.

\section{CONCLUSIONS}

A quantum model of pulsed second-subharmonic generation in a nonlinear waveguide with a periodic linear corrugation has been developed. Assuming a strong fundamental field, the model has been solved for lower second-subharmonic field intensities using perturbation approach generalized to back-scattered fields. More intense second-subharmonic fields with negligible intermode dispersion have been treated by the Fouriertransform approach that allows to find partly analytical solutions. Numerical approach has been applied in the general case. Using the Bloch-Messiah reduction spectral eigenmodes suitable for squeezed-light generation have been revealed. Scattering by the corrugation is more efficient in the fundamental field than in the secondsubharmonic one. Although scattering by the corrugation makes the second-subharmonic spectra narrower, it broadens the spectral eigenmodes. It also leads to a larger number of populated eigenmodes. Phase relations in the nonlinear interaction imposed by the corrugation also cause splitting of the temporal second-subharmonic pulse. In a sufficiently long waveguide, the corrugation dramatically increases the number of generated photons and, hand in hand, suppresses quantum amplitude fluctuations. A periodic corrugation thus represents a very important and efficient tool for tailoring properties of the light generated in modern nonlinear photonic waveguides.

\section{Appendix A: An optimum mode for the pulsed squeezed light}

We look for a suitable linear combination of the output operator amplitudes $\hat{a}_{s, i}^{\text {out }}$ that minimizes the value of principal squeeze variance $\lambda_{s}$. Using the Bloch-Messiah reduction of matrices $\mathbf{U}$ and $\mathbf{V}$ in Eq. (76) we can express 
this combination as follows [61]:

$$
\hat{a}_{s}^{\text {out }}=\sum_{i, j} t_{j} \mathbf{X}_{i j} \hat{a}_{s, i}^{\text {out }}
$$

Coefficients $t_{j}$ fulfil the normalization condition $\sum_{j}\left|t_{j}\right|^{2}=1$. Using Eqs. (48) and (49) the principal squeeze variance $\lambda_{s}^{\mathrm{L}}$ with the Lagrange term as a function of $t_{j}$ and $t_{j}^{*}$ can be expressed in the following form:

$$
\begin{aligned}
\lambda_{s}^{\mathrm{L}}\left(\mathbf{t}, \mathbf{t}^{*}\right) & =1+2 \mathbf{t}^{\dagger} \boldsymbol{\Lambda}_{V}^{2} \mathbf{t}-2\left|\mathbf{t}^{T} \boldsymbol{\Lambda}_{V} \boldsymbol{\Lambda}_{U} \mathbf{t}\right|-\mu \mathbf{t}^{\dagger} \cdot \mathbf{t}, \\
& =1+2 \mathbf{t}^{\dagger} \boldsymbol{\Lambda}_{V}^{2} \mathbf{t}-2 \mathbf{t}^{\dagger} \boldsymbol{\Lambda}_{V} \boldsymbol{\Lambda}_{U} \mathbf{t}-\mu \mathbf{t}^{\dagger} \cdot \mathbf{t} .(\mathrm{A} 2)
\end{aligned}
$$

Symbol $\mu$ in Eq. A2 denotes a Lagrange multiplier related to the normalization of vector $\mathbf{t}$ composed of coefficients $t_{j}$.

Derivation of the function $\lambda_{s}^{\mathrm{L}}$ in Eq. A2 with respect to the coefficients $t_{i}^{*}$ and $t_{i}$ gives the conditions:

$$
\begin{array}{r}
2\left[\boldsymbol{\Lambda}_{V}^{2}-\boldsymbol{\Lambda}_{V} \boldsymbol{\Lambda}_{U}\right] \mathbf{t}=\mu \mathbf{t}, \\
2 \mathbf{t}^{\dagger}\left[\boldsymbol{\Lambda}_{V}^{2}-\boldsymbol{\Lambda}_{V} \boldsymbol{\Lambda}_{U}\right]=\mu \mathbf{t}^{\dagger} .
\end{array}
$$

Assuming $\mu=2\left(\boldsymbol{\Lambda}_{V, i i}^{2}-\boldsymbol{\Lambda}_{V, i i} \boldsymbol{\Lambda}_{U, i i}\right)$ the solution to Eqs. (A3) is $t_{i}=1$ and $t_{j}=0$ for $j \neq i$. As we look for the minimum value of $\lambda_{s}$, we choose $i$ such that its principal squeeze variance given by $1+\mu$ is minimum.

\section{Acknowledgments}

The author is obliged to M. Scalora for his support and help. He also thanks A. Lukš, V. Peřinová and O. Haderka for discussions and help with analytical computations. This material is partly based upon the work supported by the European Research Office of the US Army under the Contract No. N62558-05-P-0421. J. P. thanks the COST project OC09026 and Operational Program Research and Development for Innovations - European Regional Development Fund project CZ.1.05/2.1.00/03.0058 of the Czech Ministry of Education, Youth and Sports.
[1] J. A. Armstrong, N. Bloembergen, J. Ducuing, and P. S. Pershan, Phys. Rev. 127, 1918 (1962).

[2] A. Lukš, V. Peřinová, and J. Peřina, Opt. Commun. 67, 149 (1988).

[3] J. Peřina, Quantum Statistics of Linear and Nonlinear Optical Phenomena (Kluwer, Dordrecht, 1991).

[4] L. Mandel and E. Wolf, Optical Coherence and Quantum Optics (Cambridge Univ. Press, Cambridge, 1995).

[5] V. V. Dodonov, J. Opt. B: Quantum Semiclass. Opt. 4, R1 (2002).

[6] W. Vogel and D.-G. Welsch, Lectures on Quantum Optics (Akademie Verlag, Berlin, 1994).

[7] H.-A. Bachor and T. C. Ralph, A Guide to Experiments in Quantum Optics (Wiley-VCH, Weinheim, 2004).

[8] W. Schleich and A. Wheeler, J. Opt. Soc. Am. B 4, 1715 (1987).

[9] J. Peřina and J. Bajer, Phys. Rev. A 41, 516 (1990).

[10] P. N. Batcher and D. Cotter, The Elements of Nonlinear Optics (Cambridge Univ. Press, Cambridge, 1991).

[11] R. W. Boyd, Nonlinear Optics (Academic Press, New York, 1994).

[12] M. W. Mitchell, Phys. Rev. A 79, 043835 (2009).

[13] Z. Y. Ou, Phys. Rev. A 49, 2106 (1994).

[14] M. J. Lawrence, R. L. Byer, M. M. Fejer, W. Bowen, P. K. Lam, and H.-A. Bachor, J. Opt. Soc. Am. B 19, 1592 (2002).

[15] J. Peřina Jr., Phys. Rev. A 77, 013803 (2008).

[16] P. J. Mosley, A. Christ, A. Eckstein, and C. Silberhorn, Physical Review Letters 103, 233901 (2009).

[17] M. Karpinski, C. Radzewicz, and K. Banaszek, Applied Physics Letters 94, 181105 (2009).

[18] L. Lanco, S. Ducci, J.-P. Likforman, X. Marcadet, J. A. W. van Houwelingen, H. Zbinden, G. Leo, and V. Berger, Phys. Rev. Lett. 97, 173901 (2006).

[19] J. Svozilík, M. Hendrych, A. S. Helmy, and J. P. Torres,
Opt. Express 19, 3115 (2011)

[20] R. Horn, P. Abolghasem, B. J. Bijlani, D. Kang, A. S. Helmy, and G. Weihs, Phys. Rev. Lett. 108, 153605 (2012).

[21] N. Matsuda, H. L. Jeannic, H. Fukuda, T. Tsuchizawa, W. J. Munro, K. Shimizu, K. Yamada, Y. Tokura, and H. Takesue, Sci. Rep. 2, 817 (2012).

[22] P. Dong and A. G. Kirk, Phys. Rev. Lett. 93, 133901 (2004).

[23] J. Peřina Jr. and J. Peřina, in Progress in Optics, Vol. 41, edited by E. Wolf (Elsevier, Amsterdam, 2000), pp. $361-419$.

[24] J. D. Joannopoulos, R. D. Meade, and J. N. Winn, Photonic Crystals: Molding the Flow of Light (Princeton University Press, Princeton, 1995).

[25] in Nanoscale Linear and Nonlinear Optics, AIP Vol. 560, edited by M. Bertolotti, C. M. Bowden, and C. Sibilia (AIP, Melville, 2001).

[26] J. W. Haus, R. Viswanathan, M. Scalora, A. G. Kalocsai, J. D. Cole, and J. Theimer, Phys. Rev. A 57, 2120 (1998).

[27] D. Pezzetta, C. Sibilia, M. Bertolotti, J. W. Haus, M. Scalora, M. J. Bloemer, and C. M. Bowden, J. Opt. Soc. Am. B 18, 1326 (2001).

[28] J. Peřina Jr., O. Haderka, C. Sibilia, M. Bertolotti, and M. Scalora, Phys. Rev. A 76, 033813 (2007).

[29] D. Tricca, C. Sibilia, S. Severini, M. Bertolotti, M. Scalora, C. M. Bowden, and K. Sakoda, J. Opt. Soc. Am. B 21, 671 (2004).

[30] J. Čtyroký and L. Kotačka, Opt. Quantum Electronics 32, 799 (2000).

[31] L. Kotačka and J. Čtyroký, Opt. Quantum Electronics 33, 541 (2001).

[32] D. Pezzetta, C. Sibilia, M. Bertolotti, R. Ramponi, R. Osellame, M. Marangoni, J. W. Haus, M. Scalora, M. J. Bloemer, and C. M. Bowden, J. Opt. Soc. Am. B 
19, $2102(2002)$.

[33] K. Sakoda, J. Opt. Soc. Am. B 19, 2060 (2002).

[34] J. Svozilík, M. Hendrych, and J. P. Torres, Opt. Express 20, 15015 (2012).

[35] G. Kang and A. S. Helmy, Opt. Lett. 37, 1481 (2012).

[36] J. Peřina Jr., C. Sibilia, D. Tricca, and M. Bertolotti, Phys. Rev. A 70, 043816 (2004).

[37] J. Peřina Jr., C. Sibilia, D. Tricca, and M. Bertolotti, Phys. Rev. A 71, 043813 (2005).

[38] J. Peřina Jr., M. Centini, C. Sibilia, M. Bertolotti, and M. Scalora, in Conference on Coherence and Quantum Optics (Optical Society of America, 2007), p. CSuA6.

[39] D. K. Serkland, M. M. Fejer, R. L. Byer, and Y. Yamamoto, Optics Lett. 20, 1649 (1995).

[40] D. K. Serkland, P. Kumar, M. A. Arbore, and M. M. Fejer, Optics Lett. 22, 1497 (1997).

[41] X. Yu, L. Scaccabarozzi, J. S. Harris Jr., P. S. Kuo, and M. M. Fejer, Optics Express 13, 10742 (2005).

[42] J. Huang, X. P. Xie, C. Langrock, R. V. Roussev, D. S. Hum, and M. M. Fejer, Optics Lett. 31, 604 (2006).

[43] A. W. Schober, M. Charbonneau-Lefort, and M. M. Fejer, J. Opt. Soc. Am. B 22, 1699 (2005).

[44] M. J. Werner, M. G. Raymer, M. Beck, and P. D. Drummond, Phys. Rev. A 52, 4202 (1995).

[45] P. Kumar, O. Aytur, and J. Huang, Phys. Rev. Lett. 64, 1015 (1990).

[46] J. H. Shapiro and A. Shakeel, J. Opt. Soc. Am. B 14, 232 (1997).

[47] R. S. Bennink and R. W. Boyd, Phys. Rev. A 66, 053815 (2002).
[48] S. L. Braunstein, Phys. Rev. A 71, 055801 (2005).

[49] W. Wasilewski, A. I. Lvovsky, K. Banaszek, and C. Radzewicz, Phys. Rev. A 73, 063819 (2006).

[50] A. I. Lvovsky, W. Wasilevski, and K. Banaszek, J. Mod. Opt. 54, 721 (2007).

[51] G. Patera, N. Treps, C. Fabre, and G. J. de Valcárcel, Eur. Phys. J. D 56, 123 (2010).

[52] M. Sasaki and S. Suzuki, Phys. Rev. A 73, 043807 (2006).

[53] M. Annamalai, N. Stelmakh, M. Vasilyev, and P. Kumar, Opt. Express 19, 26710 (2011).

[54] P. Scotto, Phys. Rev. A 68, 033814 (2003).

[55] M. Bache, P. Scotto, R. Zambrini, M. San Miguel, and M. Saffman, Phys. Rev. A 66, 013809 (2002).

[56] M. Scalora, M. J. Bloemer, A. S. Manka, J. P. Dowling, C. M. Bowden, R. Viswanathan, and J. W. Haus, Phys. Rev. A 56, 3166 (1997).

[57] G. D'Aguanno, M. Centini, M. Scalora, C. Sibilia, Y. Dumeige, P. Vidakovic, J. A. Levenson, M. J. Bloemer, C. M. Bowden, J. W. Haus, et al., Phys. Rev. E 64, 016609 (2001).

[58] Y. Dumeige, P. Vidakovic, S. Sauvage, I. Sagnes, J. A. Levenson, C. Sibilia, M. Centini, G. D'Aguanno, and M. Scalora, Appl. Phys. Lett. 78, 3021 (2001).

[59] J. Peřina Jr., Phys. Rev. A 84, 053840 (2011).

[60] A. Luis and J. Peřina, Quantum Semiclass. Optics 8, 39 (1996).

[61] A. Lukš, private communication.

[62] J. C. McKinstrie, Opt. Commun. 282, 583 (2009). 